PHYSICAL REVIEW D 94, 025017 (2016)

\title{
On-shell effective field theory: A systematic tool to compute power corrections to the hard thermal loops
}

\author{
Cristina Manuel* \\ Instituto de Ciencias del Espacio (IEEC/CSIC) C. Can Magrans s.n., \\ 08193 Cerdanyola del Vallès, Catalonia, Spain \\ Joan Soto \\ Departament d'Estructura i Constituents de la Matèria and Institut de Ciències del Cosmos, \\ Universitat de Barcelona, Martí i Franquès 1, 08028 Barcelona, Catalonia, Spain \\ Stephan Stetina \\ Institute for Nuclear Theory, University of Washington, Seattle, Washington 98195, USA
}

(Received 4 April 2016; published 8 July 2016)

\begin{abstract}
We show that effective field theory techniques can be efficiently used to compute power corrections to the hard thermal loops in a high temperature $T$ expansion. To this aim, we use the recently proposed onshell effective field theory, which describes the quantum fluctuations around on-shell degrees of freedom. We provide the on-shell effective field theory Lagrangian up to third order in the energy expansion for QED and use it for the computation of power corrections to the retarded photon polarization tensor for soft external momenta. Here soft denotes a scale of order $e T$, where $e$ is the gauge coupling constant. We develop the necessary techniques to perform these computations and study the contributions to the polarization tensor proportional to $e^{2} T^{2}, e^{2} T$, and $e^{2} T^{0}$. The first one describes the hard thermal loop contribution, the second one vanishes, while the third one provides corrections of order $e^{2}$ to the soft photon propagation. We check that the results agree with the direct calculation from QED, up to local pieces, as expected in an effective field theory.
\end{abstract}

DOI: $10.1103 /$ PhysRevD.94.025017

\section{INTRODUCTION}

The physics of QED and QCD plasmas at high temperature $T$ is extremely rich [1]. In the early $1990 \mathrm{~s}$, it was discovered that the soft energy and momentum scales of these plasmas, where soft denotes a scale of order $e T$ and $e$ is the gauge coupling constant, are properly described by the so-called hard thermal loop (HTL) effective field theory. HTLs were first found out by extracting from oneloop Feynman diagrams their leading behavior for soft external momenta [2-4], which arises from the contribution of the so-called hard scales (of order $T$ ) circulating in the loop. For soft scales, they are as relevant as the bare propagators or vertices of the theory, and HTLs have to be resummed. Although different derivations of the HTL diagrams were given, it was soon realized that they could be understood in terms of the classical propagation of the on-shell particles of the QED or QCD plasmas [5-7]. The HTL effective field theory has been used for a large variety of computations of both static and dynamical properties of thermal plasmas (see for example Ref. [8]), while the static properties in the high $T$ limit of QED and QCD have been

\footnotetext{
*cmanuel@ice.cat †joan.soto@ub.edu stetina@uw.edu
}

typically studied with the use of dimensional reduced effective field theories [9-11]. One of the aims of this paper is to show that effective field theory techniques can also be used to compute power corrections to the HTLs, as arising from the hard scales in the plasma.

The concept of effective field theory (EFT) is widely and successfully used in physics. It relies on the idea that in order to discuss relevant phenomena at a given energy scale, it is enough to identify the degrees of freedom that operate at that scale and uncover the Lagrangian that governs their dynamics. The Lagrangian is organized in operators of increasing dimension over powers of the high energy scales, so that all the information on the high energy scales (beyond the explicit powers) is encoded in the matching coefficients of these operators. The matching coefficients are obtained by enforcing the EFT to be equivalent to the fundamental theory at a given order in the ratio of scales and/or in some small parameter, typically a coupling constant. Nowadays, a large number of EFTs have been derived at zero temperature, from which we will only quote the ones that have been relevant for the present work. High density effective theory (HDET) describes the quantum fluctuations around the Fermi level of a finite density system, being the chemical potential the high energy scale [12]. In nonrelativistic QED/QCD (NRQED/ NRQCD) [13] the high energy scale is the mass of the heavy particles and the low energy scales the remaining ones in a 
nonrelativistic bound state. Heavy quark effective theory (HQET) [14-16] may be considered the simplest particular case of NRQCD, in which the only low energy scale is $\Lambda_{\mathrm{OCD}}$, the typical hadronic scale. The construction of HQET is formally very similar to the one of HDET, and it was a source of inspiration for the so-called large energy effective theory [17]. Soft-collinear effective theory (SCET) $[18,19]$ may be regarded as a completion of the latter, in which the high energy scale is a dynamical variable and hence the matching coefficients are dynamical functions rather than functions of fixed parameters. This feature will be shared by the EFT used in this work. It first appeared in potential NRQCD/NRQED [20], in which the quantum mechanical potentials are regarded as position-dependent matching coefficients. In recent years, some of the EFTs above have been combined with the thermal EFTs in order to study the thermal properties of nonrelativistic bound states [21-26] and jets [27].

In this manuscript, we will show that the recently proposed on-shell effective field theory (OSEFT), see Ref. [28], is a systematic and powerful tool to extract power corrections to the HTLs. As an example, we focus here in studying the retarded polarization tensor of QED. The OSEFT was used in Ref. [28] to provide a derivation of chiral kinetic theory at finite temperature [29-32]. However, its possible applications have a much wider scope. The OSEFT is meant to describe physical phenomena dominated by almost on-shell particles. Here, for simplicity, we will restrict ourselves to the case of QED with massless fermions. The formalism can be generalized to deal with on-shell massive particles or with non-Abelian interactions. Starting from the QED Lagrangian, we derive the Lagrangian describing the (small) quantum fluctuations around the on-shell degrees of freedom, which can be expanded as a series in $1 / p$, where $p$ is the energy associated to the on-shell degrees of freedom in a given frame. In a thermal plasma, for $p \sim T$ in the rest frame of the plasma, our formalism not only allows us to easily extract the HTLs but also corrections to them expanded in powers of $1 / T$. In this paper, we develop the techniques to perform these computations and extract the first corrections to the HTL associated with the retarded photon polarization tensor. We also check explicitly that the results obtained from the OSEFT agree with those obtained with full QED, at the order of accuracy in which we work, up to local counterterms, as it should be the case in an EFT.

This paper is structured as follows. In Sec. II, we present the rationale behind the OSEFT and how to derive the effective Lagrangian associated with the quantum fluctuations around on-shell particles and antiparticles. In Sec. II A, we give the explicit form of the effective Lagrangians up to order $1 / p^{3}$ in the energy expansion, after performing local field redefinitions, which facilitate the computations carried out in this manuscript. In Sec. II B, we present the propagators for the particle and antiparticle quantum fluctuations in a thermal bath, in the so-called real time formalism.
Section III is devoted to the computation of the retarded photon polarization tensor with the OSEFT. After introducing the two relevant topologies-bubble and tadpole diagrams - and making some generic comments on how to organize the calculation, we show results at order $e^{2} T^{2}$, $e^{2} T$, and $e^{2} T^{0}$, in Secs. III A, III B and III C, respectively. In Sec. III A, we obtain the standard HTL result, and in Sec. III B we show that there is no contribution at order $e^{2} T$. In Sec. III C, we present the contribution of the bubble and tadpole diagrams separately and pinpoint the inherent ambiguities of the latter at this order. We close with a discussion in Sec. IV. Appendices A and C contain technical details. Appendix B shows how the calculations can be performed in the imaginary time formalism, and in Appendix D, we carry out the calculation directly from QED in order to check the reliability of the OSEFT.

We use natural units $\hbar=c=k_{B}=1$, metric conventions $g^{\mu \nu}=(1,-1,-1,-1)$, and boldface letters to denote three-dimensional vectors.

\section{ON-SHELL EFFECTIVE FIELD THEORY}

In this section, we review how to construct the basic effective action of the OSEFT first introduced in Ref. [28]. For the computation of different physical observables dominated by the contribution of almost on-shell fermions, it is convenient to construct an EFT where the role of the quantum fluctuations is clearly singled out. Let us recall that the propagation of an on-shell massless fermion is described by its energy $E=p$, with $p>0$, and the four lightlike velocity $v^{\mu}=(1, \mathbf{v})$, where $\mathbf{v}$ is three-dimensional unit vector. Hence, for a fermion close to be on shell, its four momentum can be expressed as

$$
q^{\mu}=p v^{\mu}+k^{\mu},
$$

where $k^{\mu}$ is the residual momentum $\left(k^{\mu} \ll p\right)$, i.e. the part of the momentum which makes $q^{\mu}$ slightly off shell.

A similar decomposition of the momentum for almost on-shell antifermions can be done as follows,

$$
q^{\mu}=-p \tilde{v}^{\mu}+k^{\mu},
$$

where $\tilde{v}^{\mu}=(1,-\mathbf{v})$.

We will apply these splittings when writing the Lagrangian of almost on-shell fermions, as then

$$
\begin{aligned}
\mathcal{L} & =\sum_{p, \mathbf{v}} \mathcal{L}_{p, \mathbf{v}}, \quad \mathcal{L}_{p, \mathbf{v}}=\bar{\psi}_{\mathbf{v}} \gamma \cdot i D \psi_{\mathbf{v}}, \\
i D_{\mu} & =i \partial_{\mu}+e A_{\mu} .
\end{aligned}
$$

The electromagnetic field above is assumed to contain soft momenta only $\left(l_{\mu} \ll p\right)$. The precise meaning of the sum shown in Eq. (3) will be given later on. 
The Dirac field in Eq. (3) can be written factoring out its $p$-dependence

$$
\begin{aligned}
\psi_{\mathbf{v}}= & e^{-i p v \cdot x}\left(P_{v} \chi_{v}(x)+P_{\tilde{v}} H_{\tilde{v}}^{(1)}(x)\right) \\
& +e^{i p \tilde{v} \cdot x}\left(P_{\tilde{v}} \xi_{\tilde{v}}(x)+P_{v} H_{v}^{(2)}(x)\right),
\end{aligned}
$$

where

$$
\begin{aligned}
& P_{v}=\frac{1}{2} \gamma \cdot v \gamma_{0}, \\
& P_{\tilde{v}}=\frac{1}{2} \gamma \cdot \tilde{v} \gamma_{0}
\end{aligned}
$$

are the particle and antiparticle projectors, respectively. The fields $\chi_{v}(x)$ and $\xi_{\tilde{v}}(x)$ contain soft momenta only $\left(k_{\mu} \ll p\right)$, whereas $H_{v}^{(1)}(x)$ and $H_{v}^{(2)}(x)$ contain generic off-shell momenta. Then, after integrating out the $H_{\tilde{v}}^{(1)}$ and $H_{v}^{(2)}$ fields (see Ref. [28] for details), one obtains the following effective Lagrangian,

$$
\begin{aligned}
\mathcal{L}_{p, \mathbf{v}}= & \mathcal{L}_{p, v}+\tilde{\mathcal{L}}_{p, \tilde{v}} \\
= & \chi_{v}^{\dagger}(x)\left(i v \cdot D+i D_{\perp} \frac{1}{2 p+i \tilde{v} \cdot D} i D_{\perp}\right) \chi_{v}(x) \\
& +\xi_{\tilde{v}}^{\dagger}(x)\left(i \tilde{v} \cdot D+i D_{\perp} \frac{1}{-2 p+i v \cdot D} i D_{\perp}\right) \xi_{\tilde{v}}(x),
\end{aligned}
$$

where $D_{\perp}=P_{\perp}^{\mu \nu} \gamma_{\mu} D_{\nu}$, and

$$
P_{\perp}^{\mu \nu}=g^{\mu \nu}-\frac{1}{2}\left(v^{\mu} \tilde{v}^{\nu}+v^{\nu} \tilde{v}^{\mu}\right)
$$

is minus the transverse projector to $\mathbf{v}$, written in covariant form. Note that $D_{\perp}^{0}=0$ and, in our conventions, $k_{\perp}^{2}=P_{\perp}^{\mu \nu} k_{\mu} k_{\nu}=-\mathbf{k}_{\perp}^{2}$.

In the OSEFT, the particle and antiparticle degrees of freedom, described by the $\chi$ and $\xi$ fields, respectively, are totally decoupled. That is why the EFT techniques employed here can be seen as the quantum field theory counterpart of the Foldy-Wouthuysen diagonalization methods employed at the level of the first quantized Dirac Hamiltonian [33].

Note also that the antiparticle part of the Lagrangian keeps the same structure as the particle part, as the two are equivalent if one performs the changes $p \leftrightarrow-p$ and $v^{\mu} \leftrightarrow \tilde{v}^{\mu}$ (or $\mathbf{v} \leftrightarrow-\mathbf{v}$ ). This is a reflection of the $C P$ symmetry of the underlying theory.

\section{A. Effective Lagrangian up to third power in the energy expansion}

The effective theory just presented allows us to assess the effect of the quantum fluctuations to different processes dominated by almost on-shell fermions in an expansion in powers of $1 / p$. In order to do so, one simply has to expand in $1 / p$ the Lagrangian Eq. (7). The first two terms in this expansion were explicitly considered in Ref. [28]. They read

$$
\begin{gathered}
\mathcal{L}_{p, v}^{(0)}=\chi_{v}^{\dagger}(\mathrm{iv} \cdot D) \chi_{v}, \\
\mathcal{L}_{p, v}^{(1)}=-\frac{1}{2 p} \chi_{v}^{\dagger}\left(\boldsymbol{D}_{\perp}\right)^{2} \chi_{v}=-\frac{1}{2 p} \chi_{v}^{\dagger}\left(D_{\perp}^{2}-\frac{e}{2} \sigma_{\perp}^{\mu \nu} F_{\mu \nu}\right) \chi_{v},
\end{gathered}
$$

where $\sigma_{\perp}^{\mu \nu}=P_{\perp \alpha}^{\mu} P_{\perp \beta}^{\nu} \sigma^{\alpha \beta}$, and $\sigma^{\mu \nu}=\frac{i}{2}\left[\gamma^{\mu}, \gamma^{\nu}\right]$. We will focus on the Lagrangian for the particle fluctuations; the Lagrangian for the antiparticle fluctuations is easily obtained after performing the changes $p \leftrightarrow-p$ and $v^{\mu} \leftrightarrow \tilde{v}^{\mu}$.

The interaction terms generated at order $1 / p^{2}$, and higher, contain temporal derivatives. In order to simplify the computations of different Feynman diagrams at this and higher orders, it is convenient to perform local field redefinitions, such that only the leading order Lagrangian contains temporal derivatives acting on the fermionic fields. This is a standard procedure in nonrelativistic effective theories [34]. Thus, if at order $1 / p^{2}$ we make the field redefinition

$$
\chi_{v} \rightarrow \chi_{v}^{\prime}=\left(1+\frac{\not_{\perp}^{2}}{8 p^{2}}\right) \chi_{v},
$$

the Lagrangian at this order reads

$$
\begin{aligned}
\mathcal{L}_{p, v}^{(2)}= & \frac{1}{8 p^{2}} \chi_{v}^{\prime \dagger}\left(\left[\not_{\perp},\left[i \tilde{v} \cdot D, D_{\perp}\right]\right]\right. \\
& \left.-\left\{\left(\not_{\perp}\right)^{2},(i v \cdot D-i \tilde{v} \cdot D)\right\}\right) \chi_{v}^{\prime},
\end{aligned}
$$

where $\{$,$\} denotes the anticommutator. This Lagrangian is$ similar, though not identical, to the Lagrangian obtained for massive fermions in a nonrelativistic $1 / m$ expansion [34], with now the energy $p$ playing a similar role as the mass $m$. In the OSEFT, there is, however, an additional term proportional to $i v \cdot D-\tilde{v} \cdot D$, which is absent in NRQED in the second order correction in the mass expansion.

At order $1 / p^{3}$, a new local field redefinition eliminates the temporal derivatives at that order. Thus, after redefining

$$
\begin{aligned}
\chi_{v} \rightarrow \chi_{v}^{\prime \prime}= & \left(1-\frac{i}{8 p^{3}} D_{\perp}\left[\tilde{v} \cdot D, D_{\perp}\right]\right. \\
& \left.+\frac{i}{16 p^{3}} \not_{\perp}^{2}(v \cdot D-\tilde{v} \cdot D)-\frac{i}{16 p^{3}} \not_{\perp}^{2} \tilde{v} \cdot D\right) \chi_{v}^{\prime},
\end{aligned}
$$


one gets

$$
\begin{aligned}
\mathcal{L}_{p, v}^{(3)}= & \frac{1}{8 p^{3}} \chi_{v}^{\prime \prime \dagger}\left\{D_{\perp}^{4}+\left[D_{\perp}, i \tilde{v} \cdot D\right]^{2}\right. \\
& \left.-(i v \cdot D-i \tilde{v} \cdot D) \not_{\perp}^{2}(i v \cdot D-i \tilde{v} \cdot D)\right\} \chi_{v}^{\prime \prime} \\
& +\frac{1}{8 p^{3}} \chi_{v}^{\prime \prime \dagger}\left\{(i v \cdot D-i \tilde{v} \cdot D) D_{\perp}\left[i \tilde{v} \cdot D, D_{\perp}\right]\right. \\
& \left.-\left[i \tilde{v} \cdot D, D_{\perp}\right] D_{\perp}(i v \cdot D-i \tilde{v} \cdot D)\right\} \chi_{v}^{\prime \prime},
\end{aligned}
$$

with no dependence on temporal derivatives. Similar local field redefinitions could be done at higher orders in the energy expansion.

\section{B. Propagators of the OSEFT in a thermal bath}

In this manuscript, we will carry out computations of thermal contributions to the polarization tensor in the real time formalism (RTF), as then it is natural to split the four-momentum into an on-shell and off-shell part. In the imaginary time formalism (ITF), where the energies are written in terms of quantized Matsubara frequencies, such a splitting cannot be naturally performed. In order to present in full coherence the derivation of the fermion propagators and Feynman diagrams in the theory, we will work in the Keldysh formulation of the RTF, see Ref. [35]. However, a posteriori, it is easy to realize how similar computations can also be performed using the ITF. We defer a discussion on how those computations should be carried out to Appendix B.

In the Keldysh representation of the RTF, the propagators are formulated as $2 \times 2$ matrices, in the space spanned by particle/thermal ghosts. The fermion propagator associated with the lowest order Lagrangian $\mathcal{L}_{p, v}^{(0)}$ reads

$$
\begin{aligned}
S(k)= & P_{v} \gamma_{0}\left[\left(\begin{array}{cc}
\frac{1}{v \cdot k+i \epsilon} & 0 \\
0 & \frac{1}{v \cdot k-i \epsilon}
\end{array}\right)\right. \\
& \left.+2 \pi i \delta(v \cdot k)\left(\begin{array}{cc}
n_{f}\left(p+k_{0}\right) & n_{f}\left(p+k_{0}\right) \\
-1+n_{f}\left(p+k_{0}\right) & n_{f}\left(p+k_{0}\right)
\end{array}\right)\right],
\end{aligned}
$$

where $n_{f}(x)=1 /(\exp (|x| / T)+1)$ is the Fermi-Dirac thermal distribution function.

Equation (15) can be deduced in two different ways. The first way is to start with the Dirac fermion propagator with dependence on the full momentum $q^{\mu}$, perform the splitting of Eq. (1), keeping only the leading terms in a large $p$ expansion. Alternatively, one can deduce it from the OSEFT Lagrangian, but realizing that $p$ acts as a sort of chemical potential for the quantum fluctuations. This last observation becomes apparent when we write the Hamiltonian of the full theory in terms of $\chi_{v}, \xi_{\tilde{v}}$, and their canonical momenta. At lowest order in the energy expansion, it reads

$$
H=\sum_{p, \mathbf{v}}\left(-p \pi_{v} \chi_{v}+p \tilde{\pi}_{\tilde{v}} \xi_{\tilde{v}}+\mathcal{H}_{p, \mathbf{v}}^{(0)}\right),
$$

where the fields

$$
\pi_{v}=\frac{\partial \mathcal{L}_{p, \mathbf{v}}^{(0)}}{\partial\left(\partial^{0} \chi_{v}\right)}=i \chi_{v}^{\dagger}, \quad \tilde{\pi}_{\tilde{v}}=\frac{\partial \mathcal{L}_{p, \mathbf{v}}^{(0)}}{\partial\left(\partial^{0} \xi_{\tilde{v}}\right)}=i \xi_{\tilde{v}}^{\dagger}
$$

are the canonical conjugate fields of the $\chi_{v}$ and $\xi_{\tilde{v}}$ fields, respectively, and

$$
\mathcal{H}_{p, \mathbf{v}}^{(0)}=\pi_{v} \partial_{0} \chi_{v}+\tilde{\pi}_{\tilde{v}} \partial_{0} \xi_{\tilde{v}}-\mathcal{L}_{p, \mathbf{v}}^{(0)}
$$

is the Hamiltonian of the OSEFT.

At every order in the $1 / p$ expansion, the propagator is modified, a property that must be taken into account when performing loop computations at a given order in the energy expansion. In the remaining part of this manuscript, we will use rather the retarded, advanced, and symmetric particle propagators, which can be constructed in the Keldysh formalism in the standard way [35]:

$$
S_{R / A}=S_{11}-S_{12 / 21}, \quad S_{S}=S_{11}+S_{22} .
$$

The propagators in the Keldysh formalism as derived from considering the OSEFT Lagrangian up to order $n$ in the energy expansion read

$$
S^{R / A}(k)=\frac{P_{v} \gamma_{0}}{k_{0} \pm i \epsilon-f(\mathbf{k})},
$$

$S^{S}(k)=P_{v} \gamma_{0}\left(-2 \pi i \delta\left(k_{0}-f(\mathbf{k})\right)\left(1-2 n_{f}\left(p+k_{0}\right)\right)\right)$.

The expansion of $f(\mathbf{k})$ at order $n$ will be denoted as $f^{(n)}(\mathbf{k})$. At lowest order,

$$
f^{(0)}(\mathbf{k})=k_{\|},
$$

and we have defined $k_{\|}=\mathbf{k} \cdot \mathbf{v}$, while

$f^{(1)}(\mathbf{k})=k_{\|}+\frac{\mathbf{k}_{\perp}^{2}}{2 p}, \quad f^{(2)}(\mathbf{k})=k_{\|}+\frac{\mathbf{k}_{\perp}^{2}}{2 p}-\frac{k_{\|} \mathbf{k}_{\perp}^{2}}{2 p^{2}}$,

as follows from Eqs. (10) and (12), respectively. Note that, for convenience, we keep the propagators above unexpanded even though $f(\mathbf{k})$ contains subleading pieces in $1 / p$.

The propagators for the antiparticle quantum fluctuations can be also be easily deduced. They read

$$
\tilde{S}^{R / A}(k)=\frac{P_{\tilde{v}} \gamma_{0}}{k_{0} \pm i \epsilon-\tilde{f}(\mathbf{k})},
$$


$\tilde{S}^{S}(k)=-P_{\tilde{v}} \gamma_{0}\left(-2 \pi i \delta\left(k_{0}-\tilde{f}(\mathbf{k})\right)\left(1-2 n_{f}\left(-p+k_{0}\right)\right)\right)$,

where the function $\tilde{f}(\mathbf{k})$ can be obtained from $f(\mathbf{k})$, with the replacements $\mathbf{v} \rightarrow-\mathbf{v}$ and $p \rightarrow-p$. Note the extra minus sign in the symmetric antiparticle propagator, absent in its particle counterpart. The presence of this additional minus sign might be deduced from the full theory.

When performing computations of Feynman diagrams at a given order in the $1 / p$ expansion, the above propagators should eventually be Taylor expanded, assuming that $k_{0}, k \ll p$. However, in practice, it is more convenient to carry out the $k_{0}$ integral before performing these expansions. We will denote the pieces of this expansion as $S_{(n)}$, where $n$ labels the order of the expansion. Note also that the distribution function in the symmetric propagator must also be expanded in $k_{0}$.

Also, note that, due to the local field redefinitions introduced beyond leading order, the propagators deduced from the OSEFT and those derived from the full theory also differ beyond leading order. However, the dispersion relations coincide, as they should.

\section{COMPUTATION OF THE RETARDED PHOTON POLARIZATION TENSOR FOR SOFT MOMENTUM}

In this section, we compute in the framework of the OSEFT the one-loop retarded photon polarization tensor up to third order in the energy expansion, assuming that the photon momentum $l$ is soft, or of order $e T$. In a thermal plasma, it is well known that the leading order behavior is given by the HTL polarization tensor [3,4] (see also Ref. [36] for an alternative derivation using the RTF). As it is known that the HTLs are dominated by the contribution of almost on-shell particles and antiparticles with energies $\sim T$, we will then assume $p \sim T$. We will also assume that $l_{\mu} \ll p$, but $l_{\mu} \sim k_{\mu}$. We will effectively show that the OSEFT allows us to reproduce, to leading order, the HTL polarization tensor but also allows us to extract in a very systematic way subleading corrections to the HTLs.

There are two topologically different kinds of diagrams that contribute at one loop to the photon polarization tensor in the OSEFT, that we call bubble and tadpole diagrams, respectively. The generic form of the particle's contribution to the retarded polarization tensor for the bubble diagrams reads (see Fig. 1 left)

$$
\begin{aligned}
\Pi_{\mathrm{b}}^{\mu \nu}(l)= & -\frac{i}{2} \sum_{p, \mathbf{v}} \int \frac{d^{4} k}{(2 \pi)^{4}}\left(\operatorname{Tr}\left[V^{\mu} S_{S}(k-l) V^{\nu} S_{R}(k)\right]\right. \\
& \left.+\operatorname{Tr}\left[V^{\mu} S_{A}(k-l) V^{\nu} S_{S}(k)\right]\right) \\
& -\frac{i}{2} \sum_{p, \mathbf{v}} \int \frac{d^{4} k}{(2 \pi)^{4}}\left(\operatorname{Tr}\left[V^{\mu} S_{A}(k-l) V^{\nu} S_{A}(k)\right]\right. \\
& \left.+\operatorname{Tr}\left[V^{\mu} S_{R}(k-l) V^{\nu} S_{R}(k)\right]\right),
\end{aligned}
$$
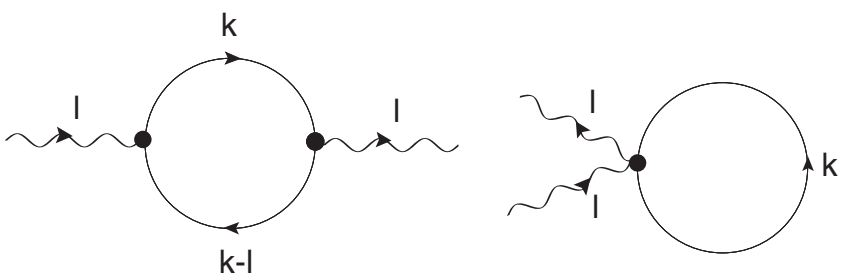

FIG. 1. We display the two topologies that contribute to the photon self-energy at one loop in the OSEFT. The blob symbolizes any vertex that may contribute to a given order.

while the tadpole diagrams are expressed as (see Fig. 1 right)

$$
\begin{aligned}
\Pi_{\mathrm{t}}^{\mu \nu}(l)= & \frac{i}{2} \sum_{p, \mathbf{v}} \int \frac{d^{4} k}{(2 \pi)^{4}} \\
& \times\left(\operatorname{Tr}\left[W^{\mu \nu}\left(S_{S}(k)+S_{R}(k)+S_{A}(k)\right)\right]\right),
\end{aligned}
$$

where we have dropped all the subindices that label the order of the energy expansion in the vertices $V^{\mu}, W^{\mu \nu}$, and in the propagators. The $k$ and $l$ dependence of $V^{\mu}$ and $W^{\mu \nu}$ must be understood. We have a single sum on $p$ and $\mathbf{v}$ above because the interaction with soft photons cannot change $p$ and $\mathbf{v}$. Further, we have taken into account that no vertex connects particle and thermal ghost propagators (i.e., $V_{12 / 21}^{\mu}=W_{12 / 21}^{\mu \nu}=0$ ).

The appearance of the tadpole diagrams in the effective field theory, which are absent in the full theory, take into account particle-photon interactions mediated by an offshell antiparticle (or vice versa for the antiparticle-photon interactions). We will see that they are necessary in order to fulfil the Ward identity $l_{\mu} \Pi^{\mu \nu}=0$ of the fundamental theory at every order in the energy expansion.

Some generic simplifications occur in the computation of both the bubble and tadpole Feynman diagrams. First, one notices that the second integral of Eq. (26) vanishes, as one can immediately check after performing the $k_{0}$ integral. This is due to the fact that the poles of the two retarded (or advanced) propagators lie on the same side of the complex plane. Similarly, one can check that terms proportional to the retarded and the advanced fermion propagator vanish in Eq. (27).

The nonvanishing terms of the bubble contribution to the retarded polarization tensor can be computed in a rather systematic and compact way thanks to the local field redefinitions introduced in Sec. II A. Feynman rules associated with the photon-fermion interactions can be extracted at every order in the $1 / p$ expansion from the Lagrangians written down in Sec. II A. The corresponding vertex appearing in the bubble diagram at order $n$ is denoted by $V_{(n)}^{\mu}$, and for completeness we present in Table I explicit values of those vertices for $n=0,1,2$. The evaluation of the bubble diagrams then requires the computation of different traces, that, in order to simplify the notation, we denote as 
TABLE I. Feynman rules for vertices involving one photon line at different orders in the energy expansion. These are derived from the Lagrangians of Eqs. (9), (10), and (12), respectively. The momentum carried out by the incoming photon is $l^{\mu}$, while $k^{\mu}$ is the momentum of the incoming fermion. We have ignored in $V_{(2)}^{\mu}$ a spindependent contribution, as it does not contribute to the bubble diagram at the order considered here. The associated Feynman rules for the antiparticles, $\tilde{V}_{(n)}^{\mu}$, are deduced from those of the particles, performing the change $p \rightarrow-p$

and $\mathbf{v} \rightarrow-\mathbf{v}$.

$V_{(0)}^{\mu}=e \gamma_{0} v^{\mu}$

$V_{(1)}^{\mu}=\frac{e}{p} \gamma_{0}\left[\left(k_{\perp}^{\mu}+\frac{1}{2} l_{\perp}^{\mu}\right)-\frac{i}{2} \sigma_{\perp}^{\mu \alpha} l_{\alpha}\right]$

$V_{(2)}^{\mu}=-\frac{e}{4 p^{2}} \gamma_{0}\left[\left(l_{\|}+2 k_{\|}\right) l_{\perp}^{\mu}+2\left(l_{\|}+2 k_{\|}\right) k_{\perp}^{\mu}+\left(\mathbf{l}_{\perp}^{2}+2 \mathbf{l}_{\perp} \cdot \mathbf{k}_{\perp}+2 \mathbf{k}_{\perp}^{2}\right) \delta^{\mu i} v^{i}+\frac{1}{2}\left((\tilde{v} \cdot l) l_{\perp}^{\mu}+\mathbf{l}_{\perp}^{2} \tilde{v}^{\mu}\right)\right]$

$$
\widehat{\operatorname{Tr}}\left[V_{(n)}^{\mu} V_{(m)}^{\nu}\right] \equiv \operatorname{Tr}\left[P_{v} \gamma_{0} V_{(n)}^{\mu} P_{v} \gamma_{0} V_{(m)}^{\nu}\right]
$$

for the particle fluctuations. In the bubble diagrams, it turns out to be convenient to defer the expansion in $1 / p$ of the propagators. Hence, we use the general form of the symmetric, retarded, and advanced propagators; perform the $k_{0}$ integral; and then expand the result in $1 / p$ at the desired order. The $k_{0}$ integral that appears in all the bubble diagrams can be easily performed, given the form of the propagators in the Keldysh representation, see Eq. (20), and also due to the fact that there is no frequency dependence in the vertices of the theory. The energy integral present in all the bubble diagrams is of the form

$$
\begin{aligned}
I_{k_{0}}= & (-2 \pi i) \frac{1}{2} \int \frac{d k_{0}}{2 \pi}\left[\left(1-2 n_{f}\left(p+k_{0}-l_{0}\right)\right)\right. \\
& \left.\times \delta\left(k_{0}-l_{0}-f(\mathbf{k}-\mathbf{l})\right) \frac{1}{k_{0}-f(\mathbf{k})+i \epsilon}\right] \\
& +(-2 \pi i) \frac{1}{2} \int \frac{d k_{0}}{2 \pi}\left[\left(1-2 n_{f}\left(p+k_{0}\right)\right) \delta\left(k_{0}-f(\mathbf{k})\right)\right. \\
& \left.\times \frac{1}{k_{0}-l_{0}-f(\mathbf{k}-\mathbf{l})-i \epsilon}\right] \\
= & \frac{-i}{l_{0}+f(\mathbf{k}-\mathbf{l})-f(\mathbf{k})+i \epsilon} \\
& \times\left[n_{f}(p+f(\mathbf{k}))-n_{f}(p+f(\mathbf{k}-\mathbf{l}))\right] .
\end{aligned}
$$

Reaching this compact formula was the main reason why we kept the propagators in (20) and (24) unexpanded.

Thus, if one wants to compute the bubble diagram at a given order in $1 / p$, one simply has to expand for large $p$ an integral like the one above, in addition to considering the possible $p$ dependence of the vertices of the diagram. In Appendix A, we present the result of these expansions as soon as the fermion dispersion law is fixed at order $1 / p^{2}$, that is, we present the explicit values of $I_{k_{0}}^{(1)}, I_{k_{0}}^{(2)}$, and $I_{k_{0}}^{(3)}$. Note that $I_{k_{0}}^{(0)}=0$, due to the fact that Eq. (29) depends on a difference of the fermion distribution functions, and $l_{\mu}$, $k_{\mu} \ll p$. If we consider the contribution to a bubble diagram with propagators at order $n$, the bubble diagram is at least one order higher in the counting, that is, it is at least of order $n+1$ in the $1 / p$ expansion.

The tadpole diagrams are very easily computed and basically only require the knowledge of the vertices $W_{(n)}^{\mu \nu}$. However, starting at order $1 / p^{3}$, they become ambiguous, even after regulating the UV divergence that appears at that order. The ambiguity amounts to local counterterms built out of the electromagnetic stress tensor, see Eq. (69), and hence it is innocuous for the consistency of the OSEFT. Nevertheless, a clear prescription must be given in order to display reproducible results. We present in Table II the value of the traces of the particle projectors times the vertices required in the computation of the polarization tensor presented in this manuscript. Since the ordering of the fields will be relevant to discuss the ambiguity, in Table II we present the results in the case that the photon with incoming momentum is to the left of the photon with outgoing momentum only. The opposite case is obtained by just changing the sign of $l$.

Note that to the above particle's tadpole and bubble contributions one should also include analogous antiparticles' contributions, $\tilde{\Pi}^{\mu \nu}{ }_{\mathrm{b}}(l)$ and $\tilde{\Pi}_{\mathrm{t}}^{\mu \nu}(l)$, which are similarly computed using the corresponding antifermion propagators and vertices. In particular, to simplify the notation, we denote

$$
\widehat{\operatorname{Tr}}\left[\tilde{V}_{(n)}^{\mu} \tilde{V}_{(m)}^{\nu}\right] \equiv \operatorname{Tr}\left[P_{\tilde{v}} \gamma_{0} \tilde{V}_{(n)}^{\mu} P_{\tilde{v}} \gamma_{0} \tilde{V}_{(m)}^{\nu}\right]
$$

the required traces for the antiparticle fluctuations. For the antiparticle fluctuations, we denote the same integral that appears in Eq. (29) by $\tilde{I}_{k_{0}}$.

In the sequel, we will present the result of the retarded polarization tensor up to $1 / p^{3}$ order, stressing again that to zero order it vanishes, $\Pi_{(0)}^{\mu \nu}=0$.

\section{A. Polarization tensor at order $e^{2} T^{2}$}

We start by computing the retarded polarization tensor at the first nontrivial order in the energy expansion. The bubble diagram can be immediately evaluated, and after performing the $k_{0}$ integral as prescribed in Eq. (29), it reads 
TABLE II. Traces needed for the computation of the tadpolelike diagrams for the particle sector. The vertices $W_{(n)}^{\mu \nu}$ involve two photon lines and are computed from Eqs. (10), (12), and (14), for the cases $n=1,2$, and 3, respectively. The momentum carried out by the incoming photon is $l^{\mu}$, while $k^{\mu}$ is the momentum of the incoming fermion. The $<$ subscript means that only the contributions corresponding to the incoming momentum carried out by the left photon are displayed. The contributions corresponding to the incoming momentum carried out by the right photon, which will be labeled by the > subscript, may be obtained by changing the sign of $l$, as displayed in the second-to-last line. The full expression reads $W_{(n)}^{\mu \nu}(k, l)$ and is displayed in the last line. Note that for $n=1$ there is no dependence on $l$ or $k$, and hence we drop them from the expressions. The corresponding expressions for the antiparticle sector may be obtained by changing $v \rightarrow \tilde{v}$ and $p \rightarrow-p$.

$$
\begin{aligned}
& \operatorname{Tr}\left(P_{v} \gamma_{0} W_{<(1)}^{\mu \nu}\right)=\frac{e^{2}}{p} P_{\perp}^{\mu \nu} \\
& \operatorname{Tr}\left(P_{v} \gamma_{0} W_{<(2)}^{\mu \nu}(k, l)\right)=\frac{e^{2}}{2 p^{2}}\left[-2 k_{\|} P_{\perp}^{\mu \nu}+\left(\left(-l_{\perp}^{\mu}+2 k_{\perp}^{\mu}\right) \delta^{\nu i}+\left(-l_{\perp}^{\nu}+2 k_{\perp}^{\nu}\right) \delta^{\mu i}\right) v^{i}\right] \\
& +\frac{1}{4 p^{3}}\left[-\tilde{v}^{\mu} l_{\perp}^{\nu}\left(\tilde{v} \cdot l-2 k_{\|}\right)-\left(8 k_{\|}-2 \tilde{v} \cdot l\right) k_{\perp}^{\mu} \delta^{\nu i} v^{i}-2 \delta^{\mu i} v^{i}\left(\left(\mathbf{l}_{\perp}^{2}-\mathbf{l}_{\perp} \mathbf{k}_{\perp}\right) \tilde{v}^{\nu}+\left(\tilde{v} \cdot l-k_{\|}\right) l_{\perp}^{\nu}\right)-2 k_{\perp}^{\mu} l_{\perp}^{\nu}+(\mu \leftrightarrow \nu)\right] \\
& \Pi_{b,(1)}^{\mu \nu}(l)+\widetilde{\Pi}_{b,(1)}^{\mu \nu}(l) \\
& =-i \sum_{p, \mathbf{v}} \int \frac{d^{3} \mathbf{k}}{(2 \pi)^{3}}\left(\widehat{\operatorname{Tr}}\left[V_{(0)}^{\mu} V_{(0)}^{\nu}\right] I_{k_{0}}^{(1)}-\widehat{\operatorname{Tr}}\left[\widetilde{V}_{(0)}^{\mu} \widetilde{V}_{(0)}^{\nu}\right] \tilde{I}_{k_{0}}^{(1)}\right),
\end{aligned}
$$$$
\operatorname{Tr}\left(P_{v} \gamma_{0} W_{<(3)}^{\mu \nu}(k, l)\right)=\frac{1}{4 p^{3}}\left[P_{\perp}^{\mu \nu}\left(-2 \mathbf{k}_{\perp}^{2}-\mathbf{l}_{\perp}^{2}+4 k_{\|}^{2}+(\tilde{v} \cdot l)^{2}-4 \tilde{v} \cdot l k_{\|}\right)+4 k_{\perp}^{\mu} k_{\perp}^{\nu}-\tilde{v}^{\mu} \tilde{v}^{\nu} \mathbf{l}_{\perp}^{2}-4 v^{i} v^{j} \delta^{\mu i} \delta^{\nu j}\left(\mathbf{l}_{\perp}^{2}+\mathbf{k}_{\perp}^{2}-2 \mathbf{l}_{\perp} \mathbf{k}_{\perp}\right)\right]
$$

where the explicit value of $I_{k_{0}}^{(1)}$ and $\tilde{I}_{k_{0}}^{(1)}$ can be found in Appendix A; see Eq. (A1). Note that the antiparticles contribute with a relative minus sign compared to the particle's contribution, due to the form of the antiparticle symmetric propagator. We then reach to

$$
\begin{aligned}
& \Pi_{b,(1)}^{\mu \nu}(l)+\widetilde{\Pi}_{b,(1)}^{\mu \nu}(l) \\
& =-2 e^{2} \sum_{p, \mathbf{v}} \int \frac{d^{3} \mathbf{k}}{(2 \pi)^{3}} \frac{d n_{f}}{d p} l_{\|}\left(\frac{v^{\mu} v^{\nu}}{v \cdot l}-\frac{\tilde{v}^{\mu} \tilde{v}^{\nu}}{\tilde{v} \cdot l}\right),
\end{aligned}
$$

where for the retarded boundary conditions $l_{0} \rightarrow l_{0}+i \epsilon$. The tadpole diagram contribution at this order is expressed as

$$
\begin{aligned}
& \Pi_{\mathrm{t},(1)}^{\mu \nu}(l)+\widetilde{\Pi}_{t,(1)}^{\mu \nu}(l) \\
& \quad=\frac{i}{2} \sum_{p, \mathbf{v}} \int \frac{d^{4} k}{(2 \pi)^{4}} \operatorname{Tr}\left[W_{(1)}^{\mu \nu} S_{S}^{(0)}(k)+\widetilde{W}_{(1)}^{\mu \nu} \widetilde{S}_{S}^{(0)}(k)\right],
\end{aligned}
$$

where the required traces at this order needed for the computation can be read in Table II. More explicitly, one finds

$$
\begin{aligned}
\Pi_{t,(1)}^{\mu \nu}(l)+\widetilde{\Pi}_{t,(1)}^{\mu \nu}(l)= & -i e^{2} \sum_{p, \mathbf{v}} \int \frac{d^{4} k}{(2 \pi)^{4}} \frac{P_{\perp}^{\mu \nu}}{p} \\
& \times(2 \pi i)\left(\delta(v \cdot k)\left(1-2 n_{f}(p)\right)\right. \\
& \left.+\delta(\tilde{v} \cdot k)\left(1-2 n_{f}(-p)\right)\right) .
\end{aligned}
$$

Note that the relative minus sign between the particle and antiparticle symmetric propagators is compensated here by the relative minus sign in the corresponding vertex for the tadpole diagram. After performing the integral on $k_{0}$, we end up with

$$
\Pi_{t,(1)}^{\mu \nu}(l)+\widetilde{\Pi}_{t,(1)}^{\mu \nu}(l)=2 e^{2} \sum_{p, \mathbf{v}} \int \frac{d^{3} \mathbf{k}}{(2 \pi)^{3}} \frac{P_{\perp}^{\mu \nu}}{p}\left(1-2 n_{f}(p)\right) .
$$

We need now to give a precise meaning to $\sum_{p, \mathbf{v}}$ in Eqs. (32) and (35). Recall that $\sum_{p, \mathbf{v}}$ together with $\int \frac{d^{3} \mathbf{k}}{(2 \pi)^{3}}$ arise from the splitting of a single variable $\mathbf{q}$ in a large component $p \mathbf{v}$ and a residual momentum $\mathbf{k}$. We should be able to reexpress the above integrands in terms of the full momemtum q, see Eq. (1), as this is the variable used in the full theory computations. If we define the quantities $k_{\|, \mathbf{q}} \equiv \mathbf{k} \cdot \hat{\mathbf{q}}$, where $\hat{\mathbf{q}}=\frac{\mathbf{q}}{q}, q=|\mathbf{q}|$, and $\mathbf{k}_{\perp, \mathbf{q}} \equiv \mathbf{k}-\hat{\mathbf{q}} k_{\|, \mathbf{q}}$, then one has to take into account that

$$
\begin{gathered}
p=q-k_{\|, \mathbf{q}}+\frac{\mathbf{k}_{\perp, \mathbf{q}}^{2}}{2 q}+\mathcal{O}\left(\frac{1}{q^{2}}\right), \\
\mathbf{v}=\hat{\mathbf{q}}-\frac{\mathbf{k}_{\perp, \mathbf{q}}}{q}-\frac{\hat{\mathbf{q}} \mathbf{k}_{\perp, \mathbf{q}}^{2}+2 k_{\|, \mathbf{q}} \mathbf{k}_{\perp, \mathbf{q}}}{2 q^{2}}+\mathcal{O}\left(\frac{1}{q^{3}}\right), \\
n_{f}(p)=n_{f}(q)+\frac{d n_{f}}{d q}\left(-k_{\|}^{\mathbf{q}}+\frac{\mathbf{k}_{\perp, \mathbf{q}}^{2}}{2 q}\right) \\
+\frac{1}{2} \frac{d^{2} n_{f}}{d q^{2}} k_{\|, \mathbf{q}}^{2}+\mathcal{O}\left(\frac{1}{q^{3}}\right) .
\end{gathered}
$$

We then use the identification (see Ref. [37]) 


$$
\sum_{p, \mathbf{v}} \int \frac{d^{3} \mathbf{k}}{(2 \pi)^{3}} \equiv \int \frac{d^{3} \mathbf{q}}{(2 \pi)^{3}}
$$

At this point, one notes that the $T=0$ contribution to the tadpole is UV divergent. We regulate such a divergence in dimensional regularization (DR), with $d=3+\epsilon$, which puts the $T=0$ contribution to zero. Then, after adding the bubble and tadpole contributions, which are needed in order that the resulting tensor respects the Ward identity, we reach to the result

$$
\begin{aligned}
\Pi_{\text {total, }(1)}^{\mu \nu}(l)= & 4 e^{2} \int \frac{d^{3} \mathbf{q}}{(2 \pi)^{3}}\left\{\frac{d n_{f}}{d q}\left(\delta^{\mu 0} \delta^{\nu 0}-l_{0} \frac{v_{\mathbf{q}}^{\mu} v_{\mathbf{q}}^{\nu}}{v_{\mathbf{q}} \cdot l}\right)\right. \\
& \left.+\mathcal{O}\left(\frac{1}{q^{2}}\right)\right\},
\end{aligned}
$$

where we have performed an integration by parts of $n_{f}$, and we have defined $v_{\mathbf{q}}^{\mu} \equiv(1, \hat{\mathbf{q}})$. We have also performed a change of variables in the contribution coming from the antiparticles, $\mathbf{v}_{\mathbf{q}} \rightarrow-\mathbf{v}_{\mathbf{q}}$, such that the antiparticle contribution can be written in the same form as the particle contribution. In this way, we reproduce to leading order the HTL polarization tensor. Note that in the result shown above we have neglected corrections of order $1 / q^{2}$. Those pieces turn out to be important when we compute higher order corrections to the polarization tensor and will be discussed in Appendix C.

For completeness, we present the explicit form of the HTL polarization tensor, that can be found out after performing the angular integrals of Eq. (40). More explicitly,

$$
\begin{aligned}
& \Pi_{\text {total },(1)}^{00}\left(l_{0}, \mathbf{l}\right)=\Pi_{\text {total },(1)}^{L}\left(l_{0}, \mathbf{l}\right), \\
& \Pi_{\text {total },(1)}^{0 i}\left(l_{0}, \mathbf{l}\right)=l_{0} \frac{l^{i}}{|\mathbf{l}|^{2}} \Pi_{\text {total, },(1)}^{L}\left(l_{0}, \mathbf{l}\right), \\
& \Pi_{\text {total },(1)}^{i j}\left(l_{0}, \mathbf{l}\right)=\left[\left(\delta^{i j}-\frac{l^{i} l^{j}}{|\mathbf{I}|^{2}}\right) \Pi_{\text {total },(1)}^{T}\left(l_{0}, \mathbf{l}\right)\right. \\
& \left.+\frac{l^{i} l^{j}}{|\mathbf{I}|^{2}} \frac{l_{0}^{2}}{|\mathbf{I}|^{2}} \Pi_{\text {total },(1)}^{L}\left(l_{0}, \mathbf{I}\right)\right],
\end{aligned}
$$

expressed in terms of the longitudinal and transverse components, given by

$$
\begin{aligned}
\Pi_{\text {total, }(1)}^{L}\left(l_{0}, \mathbf{l}\right)= & m_{D}^{2}\left(\frac { l _ { 0 } } { 2 | \mathbf { I } | } \left(\ln \left|\frac{l_{0}+|\mathbf{I}|}{l_{0}-|\mathbf{I}|}\right|\right.\right. \\
& \left.\left.-i \pi \Theta\left(|\mathbf{I}|^{2}-l_{0}^{2}\right)\right)-1\right), \\
\Pi_{\text {total },(1)}^{T}\left(l_{0}, \mathbf{l}\right)= & -m_{D}^{2} \frac{l_{0}^{2}}{2|\mathbf{I}|^{2}}\left[1+\frac{1}{2}\left(\frac{|\mathbf{I}|}{l_{0}}-\frac{l_{0}}{|\mathbf{I}|}\right)\right. \\
\times & \left.\left(\ln \left|\frac{l_{0}+|\mathbf{I}|}{l_{0}-|\mathbf{I}|}\right|-i \pi \Theta\left(|\mathbf{I}|^{2}-l_{0}^{2}\right)\right)\right],
\end{aligned}
$$

respectively. Here, $\Theta$ is the step function, and $m_{D}^{2}=\frac{e^{2} T^{2}}{6}$ is the Debye mass squared. The imaginary part of the polarization tensor gives account of Landau damping.

\section{B. Polarization tensor at order $e^{2} T$}

At this order in the expansion, we find a vanishing contribution to the polarization tensor in the rotational invariant thermal plasma. Let us explain how this happens first for the particle's contribution; the antiparticle contribution is similarly computed.

Let us consider first the tadpole diagrams, which read

$$
\begin{aligned}
\Pi_{\mathrm{t},(2)}^{\mu \nu}(l)= & \frac{i}{2} \sum_{p, \mathbf{v}} \int \frac{d^{4} k}{(2 \pi)^{4}} \\
& \times \operatorname{Tr}\left[W_{(2)}^{\mu \nu}(k, l) S_{S}^{(0)}(k)+W_{(1)}^{\mu \nu} S_{S}^{(1)}(k)\right] .
\end{aligned}
$$

The explicit expressions of the tadpole contributions at this order can be written down after using the values of the traces displayed in Table II. After expressing these contributions in terms of the original variables, it is not difficult to realize that they cancel after performing the angular integration over $\hat{\mathbf{q}}$ (note that to leading order $\mathbf{v} \sim \hat{\mathbf{q}}$ ).

A careful inspection of all the bubble diagrams that appear at this order leads to

$$
\begin{aligned}
\Pi_{b,(2)}^{\mu \nu}(l)= & -i \sum_{p, \mathbf{v}} \int \frac{d^{3} \mathbf{k}}{(2 \pi)^{3}}\left\{\left(\hat{\operatorname{Tr}}\left[V_{(1)}^{\mu} V_{(0)}^{\nu}\right]\right.\right. \\
& \left.\left.+\hat{\operatorname{Tr}}\left[V_{(0)}^{\mu} V_{(1)}^{\nu}\right]\right) I_{k_{0}}^{(1)}+\hat{\operatorname{Tr}}\left[V_{(0)}^{\mu} V_{(0)}^{\nu}\right] I_{k_{0}}^{(2)}\right\} .
\end{aligned}
$$

After using Eqs. (A1) and (A3), together with the Feynman rules of Table I, the above contribution can be expressed as

$$
\begin{aligned}
\Pi_{b,(2)}^{\mu \nu}(l)= & e^{2} \sum_{p, \mathbf{v}} \int \frac{d^{3} \mathbf{k}}{(2 \pi)^{3}}\left[\frac { 1 } { p } \frac { d n _ { f } } { d p } \left\{l_{\|}\left(l_{\perp}^{\mu} v^{\nu}+l_{\perp}^{\nu} v^{\mu}\right) \frac{1}{v \cdot l}\right.\right. \\
& \left.\left.+v^{\mu} v^{\nu}\left[\frac{\mathbf{l}_{\perp}^{2}}{v \cdot l}+\frac{\mathbf{l}_{\perp}^{2} l_{\|}}{(v \cdot l)^{2}}\right]\right\}+\frac{d^{2} n_{f}}{d p^{2}} v^{\mu} v^{\nu} \frac{l_{\|}^{2}}{(v \cdot l)}\right],
\end{aligned}
$$

where we have not written terms linear in $k_{\|}$and $\mathbf{k}_{\perp}$, as they cancel out if we assume that the formal measure of the $\mathbf{k}$-integration is invariant under $\mathbf{k} \rightarrow-\mathbf{k}$. We reexpress the value of the above integrand in terms of the original variable $\mathbf{q}$ to reach to

$$
\begin{aligned}
\Pi_{b,(2)}^{\mu \nu}(l)= & e^{2} \int \frac{d^{3} \mathbf{q}}{(2 \pi)^{3}} \frac{1}{q} \frac{d n_{f}}{d q}\left\{l_{\|, \mathbf{q}}\left(l_{\perp, \mathbf{q}}^{\mu} v_{\mathbf{q}}^{\nu}+l_{\perp, \mathbf{q}}^{\nu} v_{\mathbf{q}}^{\mu}\right) \frac{1}{v \cdot l}\right. \\
& \left.+v_{\mathbf{q}}^{\mu} v_{\mathbf{q}}^{\nu}\left(\frac{\mathbf{l}_{\perp, \mathbf{q}}^{2}-2 l_{\|, \mathbf{q}}^{2}}{v_{\mathbf{q}} \cdot l}+\frac{\mathbf{l}_{\perp, \mathbf{q}}^{2} l_{\|, \mathbf{q}}}{\left(v_{\mathbf{q}} \cdot l\right)^{2}}\right)+\mathcal{O}\left(\frac{1}{q}\right)\right\},
\end{aligned}
$$


where we have integrated by parts the fermionic distribution function. What it is most surprising, not obvious at first sight, is that Eq. (49) vanishes, after performing the angular integration.

As the antiparticle contribution at this order also vanishes, we then conclude that there is no finite contribution to the polarization tensor at order $e^{2} T$.

\section{Polarization tensor at order $e^{2} T^{0}$}

We distribute this section in two subsections. In the first one, we display the (unambiguous) contribution of the bubble diagram, and in the second one, we illustrate the inherent ambiguity of the tadpole contributions at this order by calculating them in two apparently equivalent ways. We shall focus on the contribution of particles, since the contribution of antiparticles may be easily obtained from it, as it has been done in previous sections.

\section{Bubble diagrams}

At order $1 / p^{3}$, the bubble diagrams contributing to the polarization tensor can be expressed as

$$
\begin{aligned}
\Pi_{b,(3)}^{\mu \nu}(l)= & -i \sum_{p, \mathbf{v}} \int \frac{d^{3} \mathbf{k}}{(2 \pi)^{3}} \\
& \times\left\{\widehat{\operatorname{Tr}}\left[V_{(0)}^{\mu} V_{(0)}^{\nu}\right] I_{k_{0}}^{(3)}+\widehat{\operatorname{Tr}}\left[V_{(0)}^{\mu} V_{(1)}^{\nu}+V_{(1)}^{\mu} V_{(0)}^{\nu}\right] I_{k_{0}}^{(2)}\right. \\
& \left.+\widehat{\operatorname{Tr}}\left[V_{(1)}^{\mu} V_{(1)}^{\nu}\right] I_{k_{0}}^{(1)}+\widehat{\operatorname{Tr}}\left[V_{(2)}^{\mu} V_{(0)}^{\nu}+V_{(0)}^{\mu} V_{(2)}^{\nu}\right] I_{k_{0}}^{(1)}\right\},
\end{aligned}
$$

where the needed values of the $I_{k_{0}}^{(n)}$ functions can be found in Appendix A. We note that these functions depend both linearly and quadratically on $\mathbf{k}$. However, such a dependence can be obviated, since the linear terms can be dropped, as argued before, while the quadratic terms of $I_{k_{0}}^{(3)}$ are canceled if we reexpress the contribution computed at lower orders in the $1 / p$ expansion in terms of the full momentum q. A proof of how this happens for the tadpole contribution is presented in Appendix C.

With the basic rules already explained on how to express the OSEFT loop integrals in terms of the full momentum q, we then reach

$$
\begin{aligned}
\Pi_{b,(3)}^{\mu \nu}(l)= & -2 e^{2} \int \frac{d^{3} \mathbf{q}}{(2 \pi)^{3}} \frac{1}{q^{2}} \frac{d n_{f}}{d q}\left\{\left[\frac{1}{3} \frac{l_{\|}^{3}-3 \mathbf{l}_{\perp}^{2} l_{\|}}{v \cdot l}+\frac{1}{4} \frac{\mathbf{l}_{\perp}^{4}-3 \mathbf{l}_{\perp}^{2} l_{\|}^{2}}{(v \cdot l)^{2}}+\frac{1}{4} \frac{\mathbf{l}_{\perp}^{4} l_{\|}}{(v \cdot l)^{3}}\right] v^{\mu} v^{\nu}\right. \\
& +\frac{1}{4}\left[\frac{\mathbf{l}_{\perp}^{2}-2 l_{\|}^{2}}{v \cdot l}+\frac{\mathbf{l}_{\perp}^{2} l_{\|}}{(v \cdot l)^{2}}-\frac{1}{2} \frac{l_{\|}(\tilde{v} \cdot l)}{v \cdot l}\right]\left(v^{\mu} l_{\perp}^{\nu}+v^{\nu} l_{\perp}^{\mu}\right)-\frac{1}{4} \frac{l_{\|} \mathbf{l}_{\perp}^{2}}{v \cdot l} P_{\perp}^{\mu \nu} \\
& \left.-\frac{1}{8} \frac{\mathbf{l}_{\perp}^{2} l_{\|}}{v \cdot l}\left(\tilde{v}^{\mu} v^{\nu}+\tilde{v}^{\nu} v^{\mu}\right)-\frac{1}{4} \frac{\mathbf{l}_{\perp}^{2} l_{\|}}{v \cdot l}\left(\delta^{i \mu} v^{\nu}+\delta^{i \nu} v^{\mu}\right) v^{i}+\mathcal{O}\left(\frac{1}{q}\right)\right\},
\end{aligned}
$$

where, as in previous orders, we have carried out an integration by parts in some terms in order to have the first derivative of the distribution function in all of them. Note that in order not to overcharge the notation we have dropped the subindex $\mathbf{q}$ in all the variables of the integrand above, that should be understood.

We note that the bubble contribution alone, as it happens at order $1 / p$, does not fulfil the Ward identity of QED. From Eq. (51), it is easy to see that $l_{\mu} \Pi_{b,(3)}^{\mu \nu}(l)$ contains only local terms that lead to

$$
l_{\mu} \Pi_{b,(3)}^{\mu i}\left(l_{0}, \mathbf{l}\right)=-\frac{e^{2} \mathbf{l}^{2} l^{i}}{60 \pi^{2}} \neq 0 .
$$

This can also be checked by explicitly performing the angular integrals in Eq. (51). One finds

$$
\begin{aligned}
\Pi_{b,(3)}^{00}\left(l_{0}, \mathbf{l}\right)= & \frac{e^{2}}{144 \pi^{2}}\left[16 \mathbf{l}^{2}-6 l_{0}^{2}+3 \frac{l_{0}}{|\mathbf{I}|}\left(l_{0}^{2}-3 \mathbf{l}^{2}\right)\right. \\
& \left.\times \ln \left(\frac{l_{0}+|\mathbf{I}|}{l_{0}-|\mathbf{I}|}\right)\right]
\end{aligned}
$$

and

$$
\Pi_{b,(3)}^{0 i}\left(l_{0}, \mathbf{l}\right)=\frac{l_{0} l^{i}}{|\mathbf{I}|^{2}} \Pi_{b,(3)}^{00}\left(l_{0}, \mathbf{l}\right),
$$

so that $l_{\mu} \Pi_{b,(3)}^{\mu 0}\left(l_{0}, \mathbf{l}\right)=0$. The transverse component of Eq. (51) [see the decomposition of Eqs. (43)] gives

$$
\begin{aligned}
\Pi_{b,(3)}^{T}\left(l_{0}, \mathbf{l}\right)= & \frac{e^{2}}{720 \pi^{2}}\left[-52 \mathbf{l}^{2}+70 l_{0}^{2}+30 \frac{l_{0}^{4}}{\mathbf{l}^{2}}\right. \\
& \left.-15 \frac{l_{0}^{3}}{|\mathbf{1}|^{3}}\left(l_{0}^{2}+2 \mathbf{l}^{2}-3 \frac{l^{4}}{l_{0}^{2}}\right) \ln \left(\frac{l_{0}+|\mathbf{I}|}{l_{0}-|\mathbf{l}|}\right)\right]
\end{aligned}
$$

while

$$
\frac{l_{i} l_{j}}{\mathbf{l}^{2}} \Pi_{b,(3)}^{i j}\left(l_{0}, \mathbf{l}\right)=\frac{l_{0}^{2}}{|\mathbf{l}|^{2}} \Pi_{b,(3)}^{00}\left(l_{0}, \mathbf{l}\right)+\frac{e^{2}}{2 \pi^{2}} \frac{\mathbf{l}^{2}}{30},
$$

from which we easily obtain Eq. (52). The tadpole contribution at order $1 / p^{3}$ is then required to get the Ward identity fulfilled. 
To the particle contribution, one should add the antiparticle contribution to the bubble diagram. One can show that at this order

$$
\widetilde{\Pi}_{b,(3)}^{\mu \nu}(l)=\Pi_{b,(3)}^{\mu \nu}(l) .
$$

\section{Tadpole diagrams}

In this section, we show how two apparently equivalent ways to calculate the tadpole diagrams lead to different results.

(a) Naive evaluation.-If we proceed as in the previous sections, the contribution of the tadpole diagrams reads

$$
\begin{aligned}
\Pi_{\mathrm{t},(3)}^{\mu \nu}(l)= & \frac{i}{2} \sum_{p, \mathbf{v}} \int \frac{d^{4} k}{(2 \pi)^{4}} \\
& \times \operatorname{Tr}\left[W_{(3)}^{\mu \nu}(k, l) S_{S}^{(0)}(k)+W_{(2)}^{\mu \nu}(k, l) S_{S}^{(1)}(k)\right. \\
& \left.+W_{(1)}^{\mu \nu} S_{S}^{(2)}(k)\right] .
\end{aligned}
$$

Only the first term gives a dependence on $l$. Let us evaluate it in the following. By substituting the zeroth order symmetric propagator in Eq. (58), we obtain

$\Pi_{t,(3)}^{\mu \nu}(l)=\frac{e^{2}}{2} \sum_{p, \mathbf{v}} \int \frac{d^{3} \mathbf{k}}{(2 \pi)^{3}} \operatorname{Tr}\left(P_{v} \gamma_{0} W_{(3)}^{\mu \nu}(k, l)\right)\left(1-2 n_{f}(p)\right)$.

After expressing this integral in terms of the momentum $\mathbf{q}$ according to Eq. (39), it is not difficult to check that the pure thermal contribution is IR divergent. In addition, the $T=0$ contribution is both IR and UV divergent. However, the combination that appears in Eq. (59) is IR finite, as it can be seen by expanding for small $p$ the ratio $\left(1-2 n_{f}(p)\right) / p^{3}$. So, the tadpole contribution at order $1 / p^{3}$ is logarithmically divergent in the UV, but IR finite. The UV divergent piece fulfills the Ward identity, and hence it may be canceled by adding a proper counterterm in the Lagrangian built out of the different components of the electromagnetic field strength tensor. Furthermore, finite contributions are also found, that added to Eq. (51) result in a polarization tensor which is respectful with the Ward identity.

Let us see how this effectively works. We will use DR, with $d=3+\epsilon$. We also neglect pieces that cancel after angular integration, so that the different tensorial components of Eq. (59) are

$$
\begin{gathered}
\Pi_{t,(3)}^{00}(l)=-\frac{e^{2} \mu^{3-d}}{4} \int \frac{d^{d} \mathbf{q}}{(2 \pi)^{d}} \frac{1-2 n_{f}(q)}{q^{3}} \mathbf{l}_{\perp}^{2}, \\
\Pi_{t,(3)}^{0 i}(l)=-\frac{e^{2} \mu^{3-d}}{4} \int \frac{d^{d} \mathbf{q}}{(2 \pi)^{d}} \frac{1-2 n_{f}(q)}{q^{3}} l_{0} l_{\perp}^{i},
\end{gathered}
$$

$$
\begin{aligned}
\Pi_{t,(3)}^{i j}(l)= & \frac{e^{2} \mu^{3-d}}{4} \int \frac{d^{d} \mathbf{q}}{(2 \pi)^{d}} \frac{1-2 n_{f}(q)}{q^{3}} \\
& \times\left(\left(l_{0}^{2}+l_{\|}^{2}-\mathbf{l}_{\perp}^{2}\right) P_{T}^{i j}-l_{\|}\left(l_{\perp}^{i} v^{j}+l_{\perp}^{j} v^{i}\right)-\mathbf{l}_{\perp}^{2} v^{i} v^{j}\right) .
\end{aligned}
$$

And after evaluation, these give

$$
\begin{aligned}
\Pi_{t,(3)}^{00}(l)= & \frac{e^{2}}{2 \pi^{2}} \frac{1}{4} \mathbf{l}^{2}\left(\frac{2}{3 \epsilon}+\frac{2}{3}\left(\ln \frac{\sqrt{\pi} T}{2 \mu}-\frac{\gamma}{2}-1\right)+\frac{1}{9}\right) \\
& +\mathcal{O}(\epsilon), \\
\Pi_{t,(3)}^{0 i}(l)= & \frac{e^{2}}{2 \pi^{2}} \frac{1}{4} l_{0} l^{i}\left(\frac{2}{3 \epsilon}+\frac{2}{3}\left(\ln \frac{\sqrt{\pi} T}{2 \mu}-\frac{\gamma}{2}-1\right)+\frac{1}{9}\right) \\
& +\mathcal{O}(\epsilon),
\end{aligned}
$$

where $\mu$ is the renormalization scale and $\gamma$ is Euler's constant. The longitudinal and transverse components read

$$
\begin{aligned}
& \frac{l_{i} l_{j}}{\mathbf{l}^{2}} \Pi_{t,(3)}^{i j}= \frac{e^{2}}{2 \pi^{2}} \frac{1}{4} l_{0}^{2}\left(\frac{2}{3 \epsilon}+\frac{2}{3}\left(\ln \frac{\sqrt{\pi} T}{2 \mu}-\frac{\gamma}{2}-1\right)+\frac{1}{9}\right) \\
&-\frac{e^{2}}{2 \pi^{2}} \frac{\mathbf{l}^{2}}{30}+\mathcal{O}(\epsilon), \\
&\left(\delta_{i j}-\frac{l_{i} l_{j}}{\mathbf{l}^{2}}\right) \Pi_{t,(3)}^{i j}= \frac{e^{2}}{2 \pi^{2}} \frac{1}{4} l_{0}^{2}\left(\frac{2}{3 \epsilon}+\frac{2}{3}\left(\ln \frac{\sqrt{\pi} T}{2 \mu}-\frac{\gamma}{2}-1\right)\right) \\
&+\frac{e^{2}}{2 \pi^{2}}\left(\frac{l_{0}^{2}}{36}-\frac{\mathbf{l}^{2}}{15}\right)+\mathcal{O}(\epsilon) .
\end{aligned}
$$

The antiparticle contribution to the tadpole diagrams is found to be exactly the same as the particle contribution

$$
\widetilde{\Pi}_{t,(3)}^{\mu \nu}(l)=\Pi_{t,(3)}^{\mu \nu}(l) .
$$

One can check now that the sum of the contributions of the bubble and tadpole diagrams obeys

$$
l_{\mu}\left(\Pi_{(3), b}^{\mu \nu}(l)+\Pi_{(3), t}^{\mu \nu}(l)\right)=0,
$$

and similarly, of course, for the antiparticle counterparts of these quantities.

The counterterms needed to remove the UV divergences differ from the QED vacuum ones [only the term proportional to $l_{0}^{2}$ in Eq. (65) has the same UV divergence as in QED]. We can write them as

$\mathcal{L}_{\text {c.t. }}=-\frac{Z(\alpha, \epsilon) C(\alpha, \mu)}{2} F_{0 i} F^{0 i}-\frac{Z^{\prime}(\alpha, \epsilon) C^{\prime}(\alpha, \mu)}{4} F_{i j} F^{i j}$,

where $Z$ and $Z^{\prime}$ stand for the counterterms and $C$ and $C^{\prime}$ stand for the matching coefficients, 


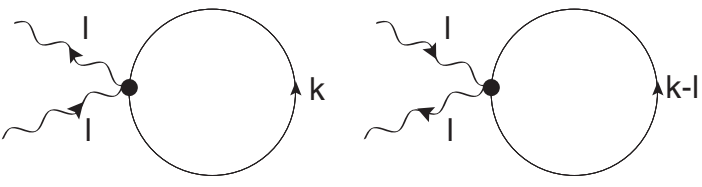

FIG. 2. This figure illustrates how the tadpole diagrams are calculated in Sec. III C 2 b.

$$
C=1+\frac{\alpha}{\pi} C^{(1)}, \quad C^{\prime}=1+\frac{\alpha}{\pi} C^{\prime(1)} .
$$

From Eqs. (63)-(66), we need in the Minimal Subtraction (MS) renormalization scheme

$$
Z=Z_{\mathrm{QED}}=1-\frac{2}{3 \epsilon} \frac{\alpha}{\pi}, Z^{\prime}=1 \neq Z_{\mathrm{QED}},
$$

and, if we compare Eq. (51) and Eqs. (63)-(66) with QED results of Appendix D, we see that they are identical if we choose

$$
C^{(1)}=0, \quad C^{\prime(1)}=\frac{2}{3}\left(\ln \frac{\sqrt{\pi} T}{2 \mu}-\frac{\gamma}{2}-1\right)+\frac{1}{9} .
$$

Whereas there is nothing wrong in the fact that the UV counterterms of the effective theory differ from the ones of the fundamental one, it is indeed somewhat surprising in our case. At one loop, the calculation in the fundamental theory involves contributions from two particle (antiparticle) legs on shell and from one particle (antiparticle) on shell and one antiparticle (particle) off shell, as it has been made explicit in Appendix D. There is a one-to-one mapping between these contributions and the bubble and tadpole diagrams of the EFT respectively. Hence, at this order in $\alpha$, the EFT appears to be exactly equivalent to the fundamental theory, and consequently one would expect the same UV behavior. The results differ because the tadpole contribution is ambiguous even in dimensional regularization. The ambiguity becomes explicit if one, for instance, puts $k-l$ rather than $k$ as the momentum in the loop. In the following section, we devise a procedure by which the UV behavior of the fundamental theory is recovered while keeping the Ward identity fulfilled.

(b) UV matched evaluation.-The rationale behind this procedure is that tadpole diagrams may be obtained from bubble diagrams in the fundamental theory by collapsing one of the legs. By doing so, one obtains one tadpole with momentum $k$ in the loop and one tadpole with momentum $k-l$ in the loop, rather than only tadpoles with momentum $k$ in the loop, as we had in Sec. III C 2 a. One prescription that provides tadpoles with momentum $k$ and $k-l$ in the loop is the following. When the incoming photon is to the left of the outgoing photon, we put $k$ as the momentum in the loop, and when it is the other way around, we put $k-l$ as the momentum in the loop; see Fig. 2. Then, formula (58) is replaced by

$$
\begin{aligned}
\Pi_{\mathrm{t},(3)}^{\mu \nu}(l)= & \Pi_{\mathrm{t},(3), \mathrm{a}}^{\mu \nu}(l)+\Pi_{\mathrm{t},(3, \mathrm{~b})}^{\mu \nu}(l)+\Pi_{\mathrm{t},(3, \mathrm{c})}^{\mu \nu}(l), \\
\Pi_{\mathrm{t},(3, \mathrm{a})}^{\mu \nu}(l)= & \frac{i}{2} \sum_{p, \mathbf{v}} \int \frac{d^{4} k}{(2 \pi)^{4}} \operatorname{Tr}\left[W_{<(3)}^{\mu \nu}(k, l) S_{S}^{(0)}(k)\right. \\
& \left.+W_{>(3)}^{\mu \nu}(k-l, l) S_{S}^{(0)}(k-l)\right], \\
\Pi_{\mathrm{t},(3, \mathrm{~b})}^{\mu \nu}(l)= & \frac{i}{2} \sum_{p, \mathbf{v}} \int \frac{d^{4} k}{(2 \pi)^{4}} \operatorname{Tr}\left[W_{<(2)}^{\mu \nu}(k, l) S_{S}^{(1)}(k)\right. \\
& \left.+W_{>(2)}^{\mu \nu}(k-l, l) S_{S}^{(1)}(k-l)\right], \\
\Pi_{\mathrm{t},(3, \mathrm{c})}^{\mu \nu}(l)= & \frac{i}{2} \sum_{p, \mathbf{v}} \int \frac{d^{4} k}{(2 \pi)^{4}} \operatorname{Tr}\left[W_{<(1)}^{\mu \nu} S_{S}^{(2)}(k)\right. \\
& \left.+W_{>(1)}^{\mu \nu} S_{S}^{(2)}(k-l)\right] .
\end{aligned}
$$

It turns out that only the pure spatial components are modified with respect to the naive prescription, so we will only provide the explicit expressions for those below:

$$
\begin{aligned}
\Pi_{t,(3, a)}^{i j}(l)= & \frac{e^{2} \mu^{3-d}}{4} \int \frac{d^{d} \mathbf{q}}{(2 \pi)^{d}} \frac{1-2 n_{f}(q)}{q^{3}} \\
& \times\left(\left(l_{0}^{2}+3 l_{\|}^{2}-2 \mathbf{l}_{\perp}^{2}\right) P_{T}^{i j}\right. \\
& \left.-3 l_{\|}\left(l_{\perp}^{i} v^{j}+l_{\perp}^{j} v^{i}\right)+\mathbf{l}_{\perp}^{2} v^{i} v^{j}\right), \\
\Pi_{\mathrm{t},(3, \mathrm{~b})}^{i j}(l)= & -\frac{e^{2}}{2} \int \frac{d^{3} \mathbf{q}}{(2 \pi)^{3}} \frac{1}{q^{2}} \frac{d n_{f}}{d q} \\
& \times\left(-2 l_{\|}^{2} P_{T}^{i j}+l_{\|}\left(l_{\perp}^{i} v^{j}+l_{\perp}^{j} v^{i}\right)\right), \\
\Pi_{\mathrm{t},(3, \mathrm{c})}^{i j}(l)= & -\frac{e^{2}}{2} \int \frac{d^{3} \mathbf{q}}{(2 \pi)^{3}} \frac{P_{T}^{i j}}{q}\left(\frac{1}{q} \frac{d n_{f}}{d q} \mathbf{l}_{\perp}^{2}+\frac{d^{2} n_{f}}{d q^{2}} l_{\|}^{2}\right) .
\end{aligned}
$$

The two last equalities above are finite and need not be dimensionally regularized like the first one. It turns out that the longitudinal component of the tadpoles is the same as the one obtained with the naive prescription Eq. (65). However, the transverse part is modified so that Eq. (66) becomes

$$
\begin{aligned}
& \left(\delta_{i j}-\frac{l_{i} l_{j}}{\mathbf{l}^{2}}\right)\left(\Pi_{\mathrm{t},(3, \mathrm{a})}^{i j}+\Pi_{\mathrm{t},(3, \mathrm{~b})}^{i j}+\Pi_{\mathrm{t},(3, \mathrm{c})}^{i j}\right) \\
& =\frac{e^{2}}{2 \pi^{2}}\left(\frac{1}{\epsilon}+\left(\ln \frac{\sqrt{\pi} T}{2 \mu}-\frac{\gamma}{2}-1\right)\right)\left(\frac{1}{6}\left(l_{0}^{2}-\mathbf{l}^{2}\right)\right) \\
& \quad+\frac{e^{2}}{2 \pi^{2}}\left(\frac{l_{0}^{2}}{36}-\frac{\mathbf{l}^{2}}{90}\right)+\mathcal{O}(\epsilon) .
\end{aligned}
$$

Antiparticles contribute in the exact same way as the particles, so to compare with the full theory, we need to multiply by 2 the above result.

As advertised, we get now the same wave function renormalization as in QED,

$$
Z=Z^{\prime}=Z_{\mathrm{QED}}=1-\frac{2}{3 \epsilon} \frac{\alpha}{\pi}
$$


However, a nonvanishing matching coefficient at order $\alpha$ is still needed to achieve agreement with the full theory result (see Appendix D)

$$
C^{(1)}=0, \quad C^{(1)}=\frac{1}{3} .
$$

\section{Final result}

We display here the final results of our calculation for the polarization tensor, which upon the inclusion of the matching coefficients $C$ and $C^{\prime}$, agree with the ones of the QED calculation of Appendix D. In the MS renormalization scheme, and for $\mu=\frac{\sqrt{\pi}}{2} T e^{-1-\gamma / 2}$, it reads

$$
\begin{aligned}
\Pi_{\text {total,(3) }}^{L}\left(l_{0}, \mathbf{l}\right)= & \frac{\alpha}{\pi}\left[\mathbf{l}^{2}-\frac{1}{3} l_{0}^{2}+\frac{1}{6} \frac{l_{0}}{|\mathbf{I}|}\left(l_{0}^{2}-3 \mathbf{l}^{2}\right)\right. \\
& \left.\times\left(\ln \left|\frac{l_{0}+|\mathbf{l}|}{l_{0}-|\mathbf{I}|}\right|-i \pi \Theta\left(|\mathbf{I}|^{2}-l_{0}^{2}\right)\right)\right], \\
\Pi_{\text {total, (3) }}^{T}\left(l_{0}, \mathbf{l}\right)= & \frac{2 \alpha}{\pi}\left[\frac{4}{9} l_{0}^{2}-\frac{43}{90} \mathbf{l}^{2}+\frac{1}{6} \frac{l_{0}^{4}}{\mathbf{l}^{2}}-\frac{1}{12} \frac{l_{0}^{3}}{|\mathbf{1}|^{3}}\right. \\
& \times\left(l_{0}^{2}+2 \mathbf{l}^{2}-3 \frac{l^{4}}{l_{0}^{2}}\right) \\
& \left.\times\left(\ln \left|\frac{l_{0}+|\mathbf{I}|}{l_{0}-|\mathbf{I}|}\right|-i \pi \Theta\left(|\mathbf{I}|^{2}-l_{0}^{2}\right)\right)\right],
\end{aligned}
$$

where we have explicitly displayed the real and imaginary parts of the polarization tensors, the last corresponding to corrections to HTL Landau damping. Let us comment here that our results of the longitudinal polarization tensor agree with the one-loop computation of $\Pi^{00}(0, \mathbf{l})$ in Ref. [38] [see Eq. (3.26)]; see also Ref. [39]. To the best of our knowledge, the complete expression of the polarization tensor at this order in the $T$ expansion has not been computed before.

\section{DISCUSSION}

We have shown how the EFT techniques that have been developed to study different systems, ranging from the high density regime to the nonrelativistic limits of QED and QCD, can also be applied to obtain power corrections to the HTLs at high temperature. We have used here the OSEFT to systematically organize the interactions of the hard scales of the plasma in powers of momenta, a fact that allows us to recognize all the contributions to the one-loop diagrams to a given order in a $1 / T$ expansion. Furthermore, with the OSEFT, we can understand the form of the nonlocalities that appear in these amplitudes at any order, as from the leading order Lagrangian Eq. (10) we see that these can only be $1 / i v$. $\partial$ or $1 / i \tilde{v}$. $\partial$ to a maximum power given by the order of the expansion [one at $\mathcal{O}\left(e^{2} T^{2}\right)$, three at $\mathcal{O}\left(e^{2} T^{0}\right)$, etc.].

Let us emphasize that the OSEFT might have many other applications than those here presented. In particular, since it properly describes the hard degrees of freedom of the plasma, it might be readily applied to the study of transport phenomena. Note also that all our basic discussion of the derivation of the EFT Lagrangian in Sec. II does not require the presence of a thermal bath, and thus the OSEFT might have applications beyond thermal field theory.

We should pinpoint here the differences and similarities that the OSEFT has with respect to other EFTs. In particular, the form of the OSEFT Lagrangian seems to be quite similar to the Lagrangian of the HDET. The main difference relies on the fact that HDET is only strictly valid at $T=0$, when there is a well-defined Fermi surface. The quantum fluctuations then are only those around the Fermi surface. The high energy scale in HDET is the chemical potential $\mu$, a fixed variable, and antiparticle fluctuations are not taken into account. In the OSEFT, the high energy scale is the dynamical on-shell energy of the particles or antiparticles, and these two degrees of freedom are treated on equal footing. HDET has been used to derive the so-called hard dense loop in Ref. [40]; the explicit meaning of the sum given in the final expressions seems to differ from the one in this paper. In that reference, the sum is over the number of patches that cover the Fermi sphere, and an explicit cutoff defining the maximal value of the residual momentum is introduced. Here, the sum is over hard momenta $p$ and the corresponding directions $\mathbf{v}$, and we can avoid the introduction of an explicit cutoff by reexpressing all the final integrals in terms of the original full momentum variable. The OSEFT also shares many similarities with SCET, the main difference being that the latter is built for a fixed number of privileged directions along which the particles are almost on shell (jetlike events), whereas in the OSEFT, the almost on-shell particles may be found in any direction.

In this manuscript, we have presented the first power correction in the high temperature expansion to the HTL polarization tensor in QED. As we already saw, the contributions to the polarization tensor at order $T$ vanish, and the first nonvanishing correction does not depend on $T$ (up to logarithms that fix the scale of the running coupling constant), even if it is due to the thermal effects in the plasma. The new correction represents modifications of order $\alpha$, the electromagnetic fine structure constant, to the soft photon propagation. This should be compared to the contributions to the photon polarization tensor arising at two-loop order from the hard scales, which are of order $e^{4} T^{2}$. Then, for soft momenta, when $l \sim e T$, the new contribution computed in this manuscript and the two-loop order result would be equally important.

Our results can be readily applied to the computation of the electromagnetic polarization tensor in the quark-gluon plasma, by just taking into account the electromagnetic charges of the different quark flavors. Again, at the QCD soft scale $l \sim g T$, where $g$ is the QCD gauge coupling constant, assumed to be small, the new contributions computed here would be of the same order as the twoloop hard contribution, and hence a leading correction to the HTL result. 
It might be worth it to compute the power corrections to the gluon polarization tensor in QCD. The quark contribution to the gluon polarization tensor could be rescued from our QED result, simply by taking into account some color and flavor factors. The gluon contribution could be computed using similar ideas to those presented here, although the proper framework to treat the gluons within the EFT should be first developed. In QCD, this would represent a next-to-leading order correction to the HTL polarization tensor (recall that in the case of QCD the soft contribution is Bose enhanced with respect to the hard one).

\section{ACKNOWLEDGMENTS}

We thank Rob Pisarski and Juan Torres-Rincon for a critical reading of our manuscript. We have been supported by the MINECO (Spain) under the CPAN CSD2007-00042 Consolider-Ingenio 2010 program and the Projects No. FPA2010-16963 and No. FPA2013-43425-P. J. S. also acknowledges the support from the Generalitat de Catalunya under Grant No. 2014-SGR-104 (Catalonia) and Project No. FPA2013-46570-C2-1-P (Spain). He has also benefited from Program No. INT-15-2c, "Equilibration Mechanisms

in Weakly and Strongly Coupled Quantum Field Theory." S.S. has been partially supported by the Schroedinger Fellowship of the FWF, Project No. J3639 (Austria).

\section{APPENDIX A: ENERGY INTEGRATION IN THE BUBBLELIKE DIAGRAMS}

In this Appendix, we present the result of the expansion in large $p$ of the integral in Eq. (29) after using the fermion dispersion law at second order; see Eq. (23). While $I_{k_{0}}^{(0)}=0$, we find

$$
\begin{gathered}
i I_{k_{0}}^{(1)}=\frac{l_{\|}}{v \cdot l} \frac{d n_{f}}{d p}, \\
i I_{k_{0}}^{(2)}=\frac{1}{2 p} \frac{d n_{f}}{d p}\left[-\frac{1}{(v \cdot l)^{2}} l_{\|}\left(\mathbf{l}_{\perp}^{2}-2 \mathbf{k}_{\perp} \cdot \mathbf{l}_{\perp}\right)\right. \\
\left.-\frac{1}{v \cdot l}\left(\mathbf{l}_{\perp}^{2}-2 \mathbf{k}_{\perp} \cdot \mathbf{l}_{\perp}\right)\right] \\
+\frac{1}{2} \frac{d^{2} n_{f}}{d p^{2}} \frac{1}{v \cdot l}\left(k_{\|}^{2}-\left(k_{\|}-l_{\|}\right)^{2}\right),
\end{gathered}
$$

$$
\begin{aligned}
i I_{k_{0}}^{(3)}= & \frac{1}{2 p^{2}} \frac{d n_{f}}{d p}\left[-\frac{1}{v \cdot l}\left(k_{\|} \mathbf{k}_{\perp}^{2}-\left(k_{\|}-l_{\|}\right)\left(\mathbf{k}_{\perp}-\mathbf{l}_{\perp}\right)^{2}\right)+\frac{1}{2} \frac{1}{(v \cdot l)^{3}} l_{\|}\left(\mathbf{l}_{\perp}^{2}-2 \mathbf{k}_{\perp} \cdot \mathbf{l}_{\perp}\right)^{2}\right. \\
& \left.+\frac{1}{2} \frac{1}{(v \cdot l)^{2}}\left(\left(\mathbf{l}_{\perp}^{2}-2 \mathbf{k}_{\perp} \cdot \mathbf{l}_{\perp}\right)^{2}+2 l_{\|}\left(\left(k_{\|}-l_{\|}\right)\left(\mathbf{k}_{\perp}-\mathbf{l}_{\perp}\right)^{2}-k_{\|} \mathbf{k}_{\perp}^{2}\right)\right)\right] \\
& +\frac{1}{2 p} \frac{d^{2} n_{f}}{d p^{2}}\left[-\frac{1}{v \cdot l}\left(\left(k_{\|}-l_{\|}\right)\left(\mathbf{k}_{\perp}-\mathbf{l}_{\perp}\right)^{2}-k_{\|} \mathbf{k}_{\perp}^{2}\right)+\frac{1}{2} \frac{1}{(v \cdot l)^{2}}\left(\mathbf{l}_{\perp}^{2}-2 \mathbf{k}_{\perp} \cdot \mathbf{l}_{\perp}\right)\left(l_{\|}^{2}-2 k_{\|} l_{\|}\right)\right] \\
& +\frac{1}{6} \frac{d^{3} n_{f}}{d p^{3}} \frac{1}{v \cdot l}\left(k_{\|}^{3}-\left(k_{\|}-l_{\|}\right)^{3}\right),
\end{aligned}
$$

and for retarded boundary conditions, $l_{0} \rightarrow l_{0}+i \epsilon$.

The same quantities defined for the antiparticles, what we call $i \tilde{I}_{k_{0}}^{(n)}$, can be deduced from the particle's counterpart, applying the basic rule of replacing $p \rightarrow-p$, and also $\frac{d}{d p} \rightarrow-\frac{d}{d p}$, and $\mathbf{v} \rightarrow-\mathbf{v}$.

\section{APPENDIX B: OSEFT COMPUTATIONS USING THE IMAGINARY TIME FORMALISM}

The computations we carried out in this manuscript using the RTF can also be reproduced using the ITF. In this Appendix, we briefly mention the main ingredients that are needed to compute the OSEFT Feynman diagrams in the ITF.

In order to proceed with the ITF, one has to perform a rotation to Euclidean space-time of the theory. One can derive the Euclidean propagators at every order in the $1 / p$ expansion from our Euclidean rotated Lagrangians, where now the energies are given by the fermionic Matsubara frequencies, $\omega_{j}=(2 j+1) \pi T$, with $j \in Z$. It is also important to realize that the energy $p$ acts as a chemical potential for the fermionic quantum fluctuations (or minus chemical potential for antifermionic fluctuations), see Eq. (16), so that the Matsubara frequencies should be shifted accordingly in the propagators.

A major simplification of the computations using the ITF is also achieved if one performs the local field redefinitions of Sec. II A. Then, the computation of the different oneloop diagrams at a given order in $1 / p$ basically involves the evaluation of two sorts of sums of Matsubara frequencies: those that appear in the bubble diagrams and those that appear in the tadpole diagrams. More particularly, for the bubble diagrams, there is always a sum over Matsubara frequencies of the form

$$
\begin{gathered}
T \sum_{j} \frac{1}{i \omega_{j}-p-f(\mathbf{k})} \frac{1}{i \omega_{j}-i \omega_{s}-p-f(\mathbf{k}-\mathbf{1})} \\
\quad=-\frac{n_{f}(p+f(\mathbf{k}-\mathbf{1}))-n_{f}(p+f(\mathbf{k}))}{-i \omega_{s}-f(\mathbf{k}-\mathbf{1})+f(\mathbf{k})},
\end{gathered}
$$

where $i \omega_{s}$ is the bosonic Matsubara frequency corresponding to the photon. Note that if we rotate back to Minkowski 
space $-i \omega_{s} \rightarrow l_{0}+i \epsilon$, we recover the result of the basic integral Eq. (29) which appears in the bubble diagrams using the RTF.

For the tadpole diagrams, the only sort of Matsubara frequency sum to be considered is

$$
T \sum_{j} \frac{1}{i \omega_{j}-p-f(\mathbf{k})}=1-2 n_{f}(p+f(\mathbf{k})),
$$

which also allows us to recover the same results for the tadpole diagrams computed with the RTF.

\section{APPENDIX C: CANCELLATION OF THE $\mathbf{k}^{2}$ TERMS}

Here, we consider only the cancellation of the pieces of order $\mathbf{k}^{2}$ in the particle contributions to the tadpoles at order $1 / p^{3}$; the same reasoning applies to the antiparticle's contribution. As these pieces are the same no matter if one computes the tadpoles using the naive prescription or the UV matched evaluation, the proof applies to these two ways of computing the tadpoles. We concentrate on the tensorial structures which are spatial.

Let us consider only the pieces which depend on $\mathbf{k}^{2}$ that appear in the computation at order $1 / p^{3}$ in the tadpole diagrams. These read

$$
\begin{aligned}
\Pi_{\mathrm{t},(3, \mathrm{a})}^{i j}(0)= & \frac{e^{2}}{2} \sum_{p, \mathbf{v}} \int \frac{d^{3} \mathbf{k}}{(2 \pi)^{3}} \frac{\left(1-2 n_{f}(p)\right)}{p^{3}} \\
& \times\left(-P_{\perp}^{i j}\left(\mathbf{k}_{\perp}^{2}-2 k_{\|}^{2}\right)+2 k_{\perp}^{i} k_{\perp}^{j}-2 v^{i} v^{j} \mathbf{k}_{\perp}^{2}\right. \\
& \left.-4 k_{\|}\left(k_{\perp}^{i} v^{j}+k_{\perp}^{j} v^{i}\right)\right), \\
\Pi_{\mathrm{t},(3, \mathrm{~b})}^{i j}(0)= & 2 e^{2} \sum_{p, \mathbf{v}} \int \frac{d^{3} \mathbf{k}}{(2 \pi)^{3}} \frac{1}{p^{2}} \\
& \times\left(-P_{\perp}^{i j} k_{\|}^{2}+k_{\|}\left(k_{\perp}^{i} v^{j}+k_{\perp}^{j} v^{i}\right)\right) \frac{d n_{f}}{d p}, \\
\Pi_{\mathrm{t},(3, \mathrm{c})}^{i j}(0)= & e^{2} \sum_{p, \mathbf{v}} \int \frac{d^{3} \mathbf{k}}{(2 \pi)^{3}} \frac{P_{\perp}^{i j}}{p}\left(-\frac{d n_{f}}{d p} \frac{\mathbf{k}_{\perp}^{2}}{p}-\frac{d^{2} n_{f}}{d p^{2}} k_{\|}^{2}\right) .
\end{aligned}
$$

These tadpole contributions can be trivially expressed in terms of the original variable $\mathbf{q}$, as to leading order $\mathbf{v} \sim \hat{\mathbf{q}}$, $n_{f}(p) \sim n_{f}(q)$, etc. We will see that all of them are cancelled by the contributions arising from the lower order tadpoles in the $1 / p$ expansion, when expressed in terms of the original momentum variable.

Let us consider the particle contribution to the tadpole diagram at order $1 / p$, and reexpress it in terms of the original momentum, keeping pieces up to order $1 / q^{3}$. More explicitly, after using Eqs. (36), (37), and (38), this tadpole diagram gives

$$
\begin{aligned}
\Pi_{\mathrm{t},(1)}^{i j}= & -e^{2} \int \frac{d^{3} \mathbf{q}}{(2 \pi)^{3}} \frac{1}{q}\left(1+\frac{k_{\|, \mathbf{q}}}{q}-\frac{\mathbf{k}_{\perp, \mathbf{q}}^{2}-2 k_{\|, \mathbf{q}}^{2}}{2 q^{2}}\right) \\
& \times\left(1-2 n_{f}(q)-2 \frac{d n_{f}}{d q}\left(-k_{\|, \mathbf{q}}+\frac{\mathbf{k}_{\perp, \mathbf{q}}^{2}}{2 q}\right)-\frac{d^{2} n_{f}}{d q^{2}} k_{\|, \mathbf{q}}^{2}\right) \\
& \times\left(\left(\delta^{i j}-\hat{\mathbf{q}}^{i} \hat{\mathbf{q}}^{j}\right)+\left(1+\frac{k_{\|, \mathbf{q}}}{q}\right) \frac{\hat{\mathbf{q}}^{i} \mathbf{k}_{\perp, \mathbf{q}}^{j}+\hat{\mathbf{q}}^{j} \mathbf{k}_{\perp, \mathbf{q}}^{i}}{q}\right. \\
& \left.+\frac{\left(\hat{\mathbf{q}}^{i} \hat{\mathbf{q}}^{j} \mathbf{k}_{\perp, \mathbf{q}}^{2}-\mathbf{k}_{\perp, \mathbf{q}}^{i} \mathbf{k}_{\perp, \mathbf{q}}^{j}\right)}{q^{2}}\right) .
\end{aligned}
$$

The pieces of order $1 / q$ give account of the particle contribution to the tadpole diagram already considered in Sec. III A. The terms of order $1 / q^{2}$ cancel after performing the angular integral. We are then left with pieces of order $1 / q^{3}$.

Even if the tadpole at order $1 / p^{2}$, Eq. (46), gives a vanishing contribution at order $e^{2} T$, it still leads-after being expressed in terms of the original variable $\mathbf{q}$ - to contributions at order $1 / q^{3}$, which have also to be considered. More particularly, Eq. (46) expressed in terms of the original variables reads

$$
\begin{aligned}
\Pi_{\mathbf{t},(2, \mathbf{a})}^{i j}(0)= & -e^{2} \int \frac{d^{3} \mathbf{q}}{(2 \pi)^{3}} \frac{1}{q^{2}}\left(1+\frac{2 k_{\|}^{\mathbf{q}}}{q}\right) \\
& \times\left(1-2 n_{f}(q)+2 k_{\|}^{\mathbf{q}} \frac{d n_{f}}{d q}\right)\left[-\left(k_{\|}^{\mathbf{q}}-\frac{\mathbf{k}_{\perp, \mathbf{q}}^{2}}{q}\right)\right. \\
& \times\left(\left(\delta^{i j}-\hat{\mathbf{q}}^{i} \hat{\mathbf{q}}^{j}\right)+\frac{\left.\hat{\mathbf{q}}^{i} \mathbf{k}_{\perp, \mathbf{q}}^{j}+\hat{\mathbf{q}}^{j} \mathbf{k}_{\perp, \mathbf{q}}^{i}\right)}{q}\right) \\
& -\left(1+\frac{k_{\|}^{\mathbf{q}}}{q}\right)\left(\hat{\mathbf{q}}^{i} \mathbf{k}_{\perp, \mathbf{q}}^{j}+\hat{\mathbf{q}}^{j} \mathbf{k}_{\perp, \mathbf{q}}^{i}\right) \\
& \left.-\frac{2\left(\hat{\mathbf{q}}^{i} \hat{\mathbf{q}}^{j} \mathbf{k}_{\perp, \mathbf{q}}^{2}-\mathbf{k}_{\perp, \mathbf{q}}^{i} \mathbf{k}_{\perp, \mathbf{q}}^{j}\right)}{q}\right], \\
\Pi_{\mathrm{t},(2, \mathbf{b})}^{i j}(0)= & 2 e^{2} \int \frac{d^{3} \mathbf{q}}{(2 \pi)^{3}} \frac{1}{q}\left(1+\frac{k_{\|}^{\mathbf{q}}}{q}\right)\left(\frac{d n_{f}}{d q}\left(k_{\|}^{\mathbf{q}}-\frac{\mathbf{k}_{\perp, \mathbf{q}}^{2}}{q}\right)\right. \\
& \left.-\frac{d^{2} n_{f}}{d p^{2}} k_{\|, \mathbf{q}}^{2}\right)\left[\left(\delta^{i j}-\hat{\mathbf{q}}^{i} \hat{\mathbf{q}}^{j}\right)+\frac{\hat{\mathbf{q}}^{i} \mathbf{k}_{\perp, \mathbf{q}}^{j}+\hat{\mathbf{q}}^{j} \mathbf{k}_{\perp, \mathbf{q}}^{i}}{q}\right],
\end{aligned}
$$

which correspond to the first and second terms of Eq. (46), respectively. As mentioned in Sec. III B, the pieces of order $1 / q^{2}$ above cancel after performing the angular integral.

It is now easy to see that the sum of all the tadpole contributions at order $1 / q^{3}$, Eqs. (C1) to (C6), leads to a cancellation of the $\mathbf{k}^{2}$ dependence at this order.

Similar computations should be carried out to see that the $\mathbf{k}^{2}$ pieces in the bubble diagrams which appear at order $1 / p^{3}$ cancel when reexpressing the bubble contribution 
computed at lower orders in the $1 / p$ expansion in terms of $\mathbf{q}$.

\section{APPENDIX D: RETARDED POLARIZATION TENSOR IN QED}

In this Appendix, we present the computation of the retarded polarization tensor in QED for soft external momentum $\sim e T$, and at the same order of accuracy that was computed in this paper. We also use the RTF and analyze and compare the result with that obtained with the OSEFT. Let us recall that to leading order in a $T$ expansion one obtains the HTL, and that follows upon expanding the value of the integrand of the polarization tensor for large values of the loop momentum, which is assumed to be of order $T$. Subleading terms in the $T$ expansion of the polarization tensor can be obtained as well if one keeps subleading terms in the expansion of the integrand. This is the computation we have carried out to verify the validity of our OSEFT results and that we briefly summarize here.

In QED, the retarded photon polarization tensor in the RTF reads [36]

$$
\begin{aligned}
\Pi^{\mu \nu}(l)= & -\frac{i e^{2}}{2} \int \frac{d^{4} q}{(2 \pi)^{4}}\left(\operatorname{Tr}\left[\gamma^{\mu} S_{S}\left(q^{\prime}\right) \gamma^{\nu} S_{R}(q)\right]\right. \\
& \left.+\operatorname{Tr}\left[\gamma^{\mu} S_{A}\left(q^{\prime}\right) \gamma^{\nu} S_{S}(q)\right]\right) \\
& -\frac{i e^{2}}{2} \int \frac{d^{4} q}{(2 \pi)^{4}}\left(\operatorname{Tr}\left[\gamma^{\mu} S_{A}\left(q^{\prime}\right) \gamma^{\nu} S_{A}(q)\right]\right. \\
& \left.+\operatorname{Tr}\left[\gamma^{\mu} S_{R}\left(q^{\prime}\right) \gamma^{\nu} S_{R}(q)\right]\right),
\end{aligned}
$$

where $q^{\prime}=q-l$ and $S_{S}(q)$ and $S_{R / A}(q)$ are the electron propagators

$$
\begin{aligned}
S_{R / A}(q) & =\frac{\gamma \cdot q}{q^{2} \pm i \operatorname{sgn}\left(q_{0}\right) \epsilon} \\
S_{S}(q) & =-2 \pi i \gamma \cdot q\left(1-2 n_{f}\left(q_{0}\right)\right) \delta\left(q^{2}\right)
\end{aligned}
$$

and contain both the particle and antiparticle degrees of freedom.

The trace is easily evaluated,

$$
\begin{aligned}
J^{\mu \nu}[q ; l] & \equiv \operatorname{Tr}\left[\gamma^{\mu}\left(\gamma \cdot q^{\prime}\right) \gamma^{\nu}(\gamma \cdot q)\right] \\
& =4\left[q^{\mu} q^{\prime \nu}+q^{\prime \mu} q^{\nu}-g^{\mu \nu} q \cdot q^{\prime}\right] .
\end{aligned}
$$

The $q_{0}$-integration performed on the second integral of Eq. (D1) reduces to zero, as one can always close the contour in a half-plane that does not contain a pole. We then consider the first term of Eq. (D1),

$$
\begin{aligned}
\operatorname{Tr}\left[\gamma^{\mu}\right. & \left.S_{S}\left(q^{\prime}\right) \gamma^{\nu} S_{R}(q)\right] \\
= & -8 \pi i\left(1-2 n_{f}\left(q_{0}^{\prime}\right)\right) \delta\left(q^{\prime 2}\right) \\
& \times \frac{1}{q^{2}+i \operatorname{sign}\left(q_{0}\right) \epsilon} J^{\mu \nu}[q ; l] .
\end{aligned}
$$

The denominator and delta function can be decomposed in the following manner:

$$
\begin{aligned}
& \frac{1}{q_{0}^{2}-\mathbf{q}^{2}+i \operatorname{sign}\left(q_{0}\right) \epsilon} \\
&=\frac{1}{2|\mathbf{q}|}\left(\frac{1}{q_{0}-|\mathbf{q}|+i \epsilon}-\frac{1}{q_{0}+|\mathbf{q}|+i \epsilon}\right), \\
& \delta\left(q^{\prime 2}\right)= \frac{1}{2|\mathbf{q}-\mathbf{l}|}\left\{\delta\left[q_{0}-\left(l_{0}+|\mathbf{q}-\mathbf{l}|\right)\right]\right. \\
&\left.+\delta\left[q_{0}-\left(l_{0}-|\mathbf{q}-\mathbf{l}|\right)\right]\right\} .
\end{aligned}
$$

These decompositions allow us to clearly identify the particle-particle, antiparticle-antiparticle, and mixed contributions to the polarization tensor, a step that help us in our comparison with the OSEFT. We then arrive at

$$
\begin{aligned}
\operatorname{Tr}\left[\gamma^{\mu} S_{S}\left(q^{\prime}\right) \gamma^{\nu} S_{R}(q)\right]= & -2 i \pi \frac{\left(1-2 n_{f}(|\mathbf{q}-\mathbf{l}|)\right)}{|\mathbf{q}||\mathbf{q}-\mathbf{l}|}\left(\left.\left[\frac{1}{l_{0}+|\mathbf{q}-\mathbf{l}|-|\mathbf{q}|+i \epsilon}-\frac{1}{l_{0}+|\mathbf{q}-\mathbf{l}|+|\mathbf{q}|+i \epsilon}\right] J^{\mu \nu}\right|_{q_{0}=l_{0}+|\mathbf{q}-\mathbf{l}|}\right. \\
& \left.+\left.\left[\frac{1}{l_{0}-|\mathbf{q}-\mathbf{l}|-|\mathbf{q}|-i \epsilon}-\frac{1}{l_{0}-|\mathbf{q}-\mathbf{l}|+|\mathbf{q}|-i \epsilon}\right] J^{\mu \nu}\right|_{q_{0}=l_{0}-|\mathbf{q}-\mathbf{l}|}\right) .
\end{aligned}
$$

Observe that each component of $J^{\mu \nu}$ depends on $q_{0}$ and is therefore modified by the delta function of the symmetric propagator in a different way, according to whether one considers the contribution of an on-shell particle or antiparticle. A similar calculation has to be performed for the second term of Eq. (D1), which gives the contribution of on-shell particles and antiparticles carrying momentum $\mathbf{q}$ rather than $\mathbf{q}-\mathbf{l}$ as above. The fact that both $\mathbf{q}$ and $\mathbf{q}-\mathbf{l}$ on-shell momenta appear in the QED calculation suggests the prescription used in Sec. III C 2 b to compute the tadpole diagrams in the OSEFT. Then, one expands the resulting expressions for large $|\mathbf{q}|$. At leading order $\left(\mathcal{O}\left(e^{2} T^{2}\right)\right.$ ), one obtains the HTL result. At $\mathcal{O}\left(e^{2} T^{1}\right)$, the expressions can still be handled analytically and lead to the same result as provided by the OSEFT in Eq. (49), which cancels after performing the angular integral. At $\mathcal{O}\left(e^{2} T^{0}\right)$, 
there are a large number of terms in the expansion, and we have carried out such a computation with the aid of a computer algebra system (Mathematica).

While at the lowest orders in the computation the structure of the bubble and tadpole diagrams that we encounter in the OSEFT is clearly seen, at order $e^{2} T^{0}$, the comparison with the OSEFT computation is not so straightforward. In order to reproduce the OSEFT structure of terms (that is, the same sort of integrals that appear in both the bubble and tadpole diagrams) within QED, angular integrations have to be carried out, and also one has to integrate by parts the Fermi distribution function. This applies to all orders in the $T$ expansion, but at this order, things are more subtle. This is in part due to the local field redefinitions we performed at order $1 / p^{2}$ in the OSEFT to simplify the computations, which are clearly manifested at this order, and also to the appearance of logarithmic UV divergences. For instance, if we call "tadpole" in QED those pieces of which the integrand is proportional to $\left(1-2 n_{f}(q)\right)$, we see that there are also contributions arising from particle-particle and antiparticle-antiparticle interactions, and not only from mixed particle-antiparticle terms as it happens in the OSEFT. Let us call the remaining contributions "bubble," which upon partial integrations become proportional to $d n_{f}(q) / d q$; we then have

$$
\Pi^{\mu \nu}(l)=\Pi_{t^{\prime}}^{\mu \nu}(l)+\Pi_{b^{\prime}}^{\mu \nu}(l) .
$$

The spatial components of the tadpole contribution read

$$
\begin{aligned}
\Pi_{t^{\prime}}^{i j}(l)= & -\mu^{3-d} \int \frac{d^{d} \mathbf{q}}{(2 \pi)^{d}}\left\{\frac { ( 1 - 2 n _ { f } ) } { 2 | \mathbf { q } | ^ { 3 } } \left[3 l_{\|}\left(l^{i} v^{j}+v^{j} l^{i}\right)\right.\right. \\
& \left.\left.-\left(l_{0}^{2}+7 l_{\|}^{2}-3 l_{\perp}^{2}\right) v^{i} v^{j}+\left(l_{0}^{2}+l_{\|}^{2}-2 l_{\perp}^{2}\right) \delta^{i j}\right]\right\} .
\end{aligned}
$$

As in the OSEFT, this expression is UV divergent, and it is regularized using DR, providing the photon wave function renormalization, as well as other finite contributions. The UV divergent terms agree with the ones obtained in Sec. III C 2 b but disagree with the ones of Sec. III C 2 a, as remarked before. After regularization, part of the finite contributions can then also be expressed as a contribution proportional to the derivative of the Fermi distribution function, and hence of the form of the local pieces that may arise in the bubble contribution. The spatial components of the bubble contribution in QED read

$$
\begin{aligned}
\Pi_{b^{\prime}}^{i j}(l)= & -\frac{e^{2}}{2} \int \frac{d^{3} \mathbf{q}}{(2 \pi)^{3}} \frac{1}{q^{2}} \frac{d n_{f}}{d q}\left(\left[2 l_{\|}+\left(l_{\perp}^{2}-3 l_{\|}^{2}\right)\left(\frac{1}{v \cdot l}-\frac{1}{\tilde{v} \cdot l}\right)\right.\right. \\
& \left.+l_{\perp}^{2} l_{\|}\left(\frac{1}{(v \cdot l)^{2}}+\frac{1}{(\tilde{v} \cdot l)^{2}}\right)\right]\left(l^{i} v^{j}+v^{j} l^{i}\right)+\left[2 l_{\perp}^{2}-8 l_{\|}^{2}-\left(8 l_{\perp}^{2} l_{\|}-\frac{22}{3} l_{\|}^{3}\right)\right. \\
& \left.\times\left(\frac{1}{v \cdot l}-\frac{1}{\tilde{v} \cdot l}\right)+\left(l_{\perp}^{4}-5 l_{\|}^{2} l_{\perp}^{2}\right)\left(\frac{1}{(v \cdot l)^{2}}+\frac{1}{(\tilde{v} \cdot l)^{2}}\right)+l_{\perp}^{4} l_{\|}\left(\frac{1}{(v \cdot l)^{3}}-\frac{1}{(\tilde{v} \cdot l)^{3}}\right)\right] v^{i} v^{j} \\
& \left.+\left[4 l_{\|}^{2}-2 l_{\perp}^{2}+l_{\|} l_{\perp}^{2}\left(\frac{1}{v \cdot l}-\frac{1}{\tilde{v} \cdot l}\right)\right] \delta^{i j}\right)
\end{aligned}
$$

where the particle and antiparticle contributions are displayed. The latter, after performing the change of variables $\mathbf{v} \rightarrow-\mathbf{v}$ in the integral, can be expressed in the same way as the particle contribution. The nonlocal pieces of the above expression agree with twice the nonlocal pieces of Eq. (51), whereas the local pieces above add up to zero upon angular integration.

For $\Pi^{00}(l)$ and $\Pi^{0 i}(l)$, we obtain exactly the same expressions as in Sec. III C both for the bubble and tadpole contributions and also for UV divergent and finite pieces.
In summary, we have checked that the OSEFT reproduces the polarization tensor of QED, up to a local piece at order $e^{2} T^{0}$. This requires the addition of the electric and magnetic terms of the Maxwell Lagrangian multiplied by suitable matching coefficients, as discussed in Secs. III C 2 a and III C 2 b. The final result for the longitudinal and transverse components of the polarization tensor is displayed in Eqs. (78) and (79). 
[1] J. P. Blaizot and E. Iancu, Phys. Rep. 359, 355 (2002).

[2] R. D. Pisarski, Phys. Rev. Lett. 63, 1129 (1989).

[3] E. Braaten and R. D. Pisarski, Nucl. Phys. B337, 569 (1990).

[4] J. Frenkel and J. C. Taylor, Nucl. Phys. B334, 199 (1990).

[5] V. P. Silin, Zh. Eksp. Teor. Fiz. 38, 1577 (1960) [Sov. Phys. JETP 11, 1136 (1960)].

[6] P. F. Kelly, Q. Liu, C. Lucchesi, and C. Manuel, Phys. Rev. Lett. 72, 3461 (1994).

[7] P. F. Kelly, Q. Liu, C. Lucchesi, and C. Manuel, Phys. Rev. D 50, 4209 (1994).

[8] N. Haque, A. Bandyopadhyay, J. O. Andersen, M. G. Mustafa, M. Strickland, and N. Su, J. High Energy Phys. 05 (2014) 027.

[9] T. Appelquist and R. D. Pisarski, Phys. Rev. D 23, 2305 (1981).

[10] K. Kajantie, M. Laine, K. Rummukainen, and M. E. Shaposhnikov, Nucl. Phys. B458, 90 (1996).

[11] E. Braaten and A. Nieto, Phys. Rev. D 51, 6990 (1995).

[12] D. K. Hong, Phys. Lett. B 473, 118 (2000).

[13] W. E. Caswell and G. P. Lepage, Phys. Lett. B 167, 437 (1986).

[14] E. Eichten and B. R. Hill, Phys. Lett. B 234, 511 (1990).

[15] H. Georgi, Phys. Lett. B 240, 447 (1990).

[16] B. Grinstein, Nucl. Phys. B339, 253 (1990).

[17] M. J. Dugan and B. Grinstein, Phys. Lett. B 255, 583 (1991).

[18] C. W. Bauer, S. Fleming, and M. E. Luke, Phys. Rev. D 63, 014006 (2000).

[19] C. W. Bauer, S. Fleming, D. Pirjol, and I. W. Stewart, Phys. Rev. D 63, 114020 (2001).

[20] A. Pineda and J. Soto, Nucl. Phys. B, Proc. Suppl. 64, 428 (1998),

[21] M. A. Escobedo and J. Soto, Phys. Rev. A 78, 032520 (2008).
[22] N. Brambilla, J. Ghiglieri, A. Vairo, and P. Petreczky, Phys. Rev. D 78, 014017 (2008).

[23] N. Brambilla, M. A. Escobedo, J. Ghiglieri, J. Soto, and A. Vairo, J. High Energy Phys. 09 (2010) 038.

[24] M. A. Escobedo and J. Soto, Phys. Rev. A 82, 042506 (2010).

[25] M. A. Escobedo, J. Soto, and M. Mannarelli, Phys. Rev. D 84, 016008 (2011).

[26] M. A. Escobedo, F. Giannuzzi, M. Mannarelli, and J. Soto, Phys. Rev. D 87, 114005 (2013).

[27] M. Benzke, N. Brambilla, M. A. Escobedo, and A. Vairo, J. High Energy Phys. 02 (2013) 129.

[28] C. Manuel and J. M. Torres-Rincon, Phys. Rev. D 90, 076007 (2014).

[29] D. T. Son and N. Yamamoto, Phys. Rev. Lett. 109, 181602 (2012).

[30] M. A. Stephanov and Y. Yin, Phys. Rev. Lett. 109, 162001 (2012).

[31] D. T. Son and N. Yamamoto, Phys. Rev. D 87, 085016 (2013).

[32] C. Manuel and J. M. Torres-Rincon, Phys. Rev. D 89, 096002 (2014).

[33] L. L. Foldy and S. A. Wouthuysen, Phys. Rev. 78, 29 (1950).

[34] A. V. Manohar, Phys. Rev. D 56, 230 (1997).

[35] K. c. Chou, Z. b. Su, B. 1. Hao, and L. Yu, Phys. Rep. 118, 1 (1985).

[36] M. E. Carrington, D. f. Hou, and M. H. Thoma, Eur. Phys. J. C 7, 347 (1999).

[37] M. E. Luke, A. V. Manohar, and I. Z. Rothstein, Phys. Rev. D 61, 074025 (2000).

[38] J. P. Blaizot, E. Iancu, and R. R. Parwani, Phys. Rev. D 52, 2543 (1995).

[39] J. O. Andersen, Phys. Rev. D 53, 7286 (1996).

[40] T. Schafer, Nucl. Phys. A728, 251 (2003). 